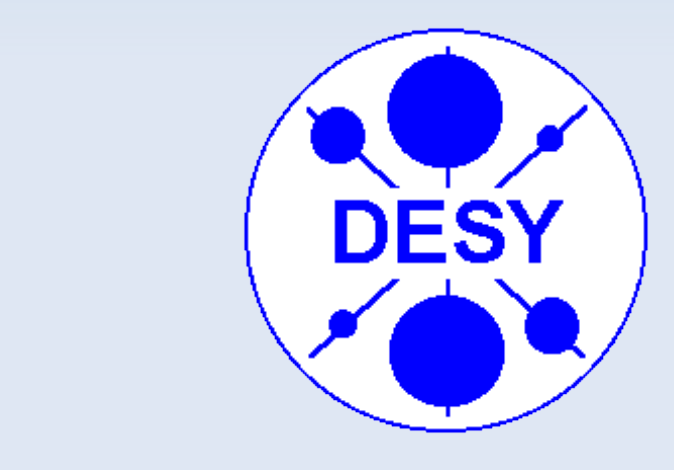

Mainz, August 7, 2014

review: arXiv:1302.6713

\title{
Quantum transport and electroweak
baryogenesis Quantum transport and electroweak
baryogenesis \\ Quantum transport and electroweak
baryogenesis
}

\author{
2014
6713
}

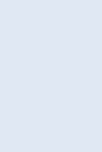

.

\section{(1)}

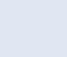

\section{Thomas Konstandin

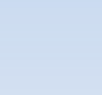

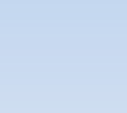

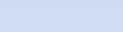

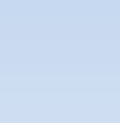

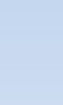

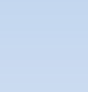
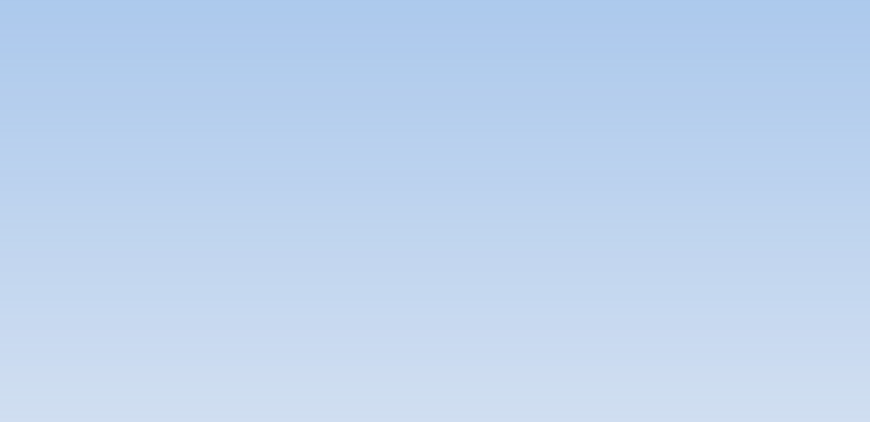

(1)

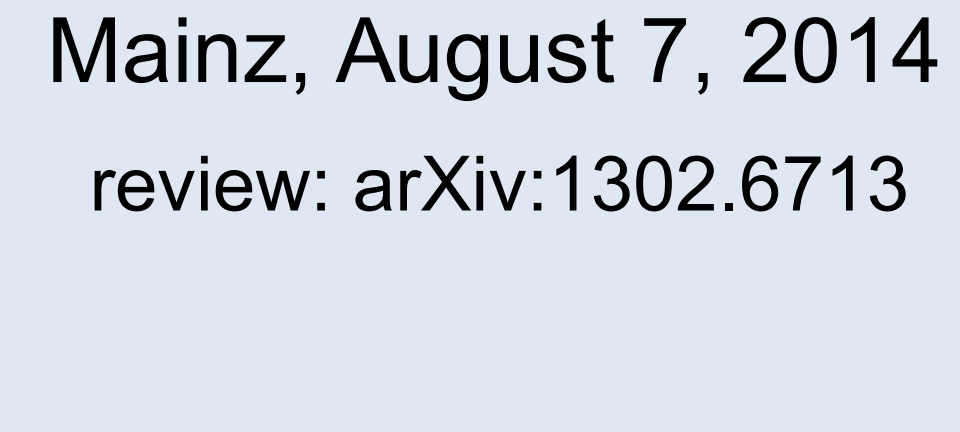

Mai

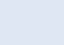

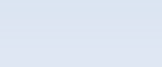

.

(1)

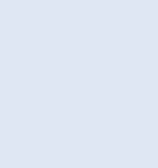

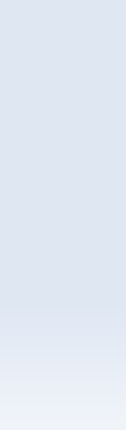

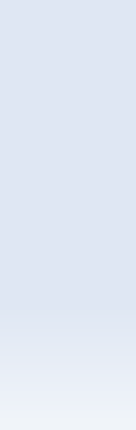
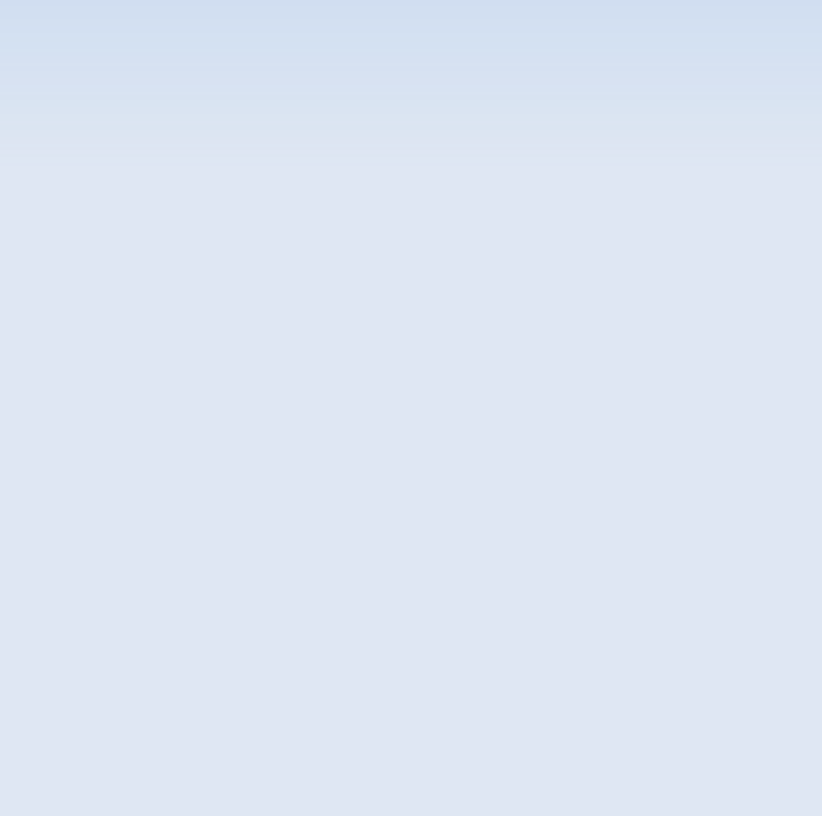

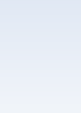




\section{Outline}

\section{Introduction}

MSSM

Composite Higgs 


\section{Baryogenesis}

\section{[Sakharov '69]}

Baryogenesis aims at explaining the observed asymmetry between matter and antimatter abundances.

$$
\eta=\frac{n_{B}-n_{\bar{B}}}{n_{\gamma}} \simeq 10^{-10}
$$

The main ingredients for viable baryogenesis are stated by the celebrated Sakharov conditions:

- B-number violation (baryon-number)

- $\mathrm{C}$ and $\mathrm{CP}$ violation (charge/parity)

- out-of-equilibrium 


\section{Baryogenesis}

\section{[Sakharov '69]}

Baryogenesis aims at explaining the observed asymmetry between matter and antimatter abundances.

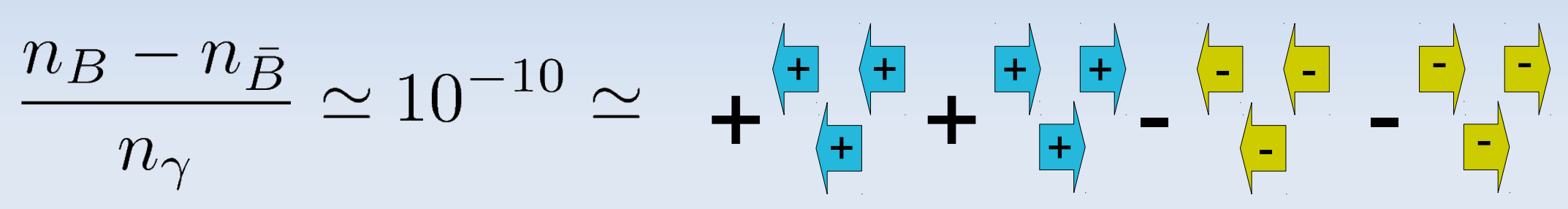

The main ingredients for viable baryogenesis are stated by the celebrated Sakharov conditions:

- B-number violation (baryon-number)

- $\mathrm{C}$ and $\mathrm{CP}$ violation (charge/parity)

- out-of-equilibrium 


\section{Baryogenesis}

\section{[Sakharov '69]}

Baryogenesis aims at explaining the observed asymmetry between matter and antimatter abundances.

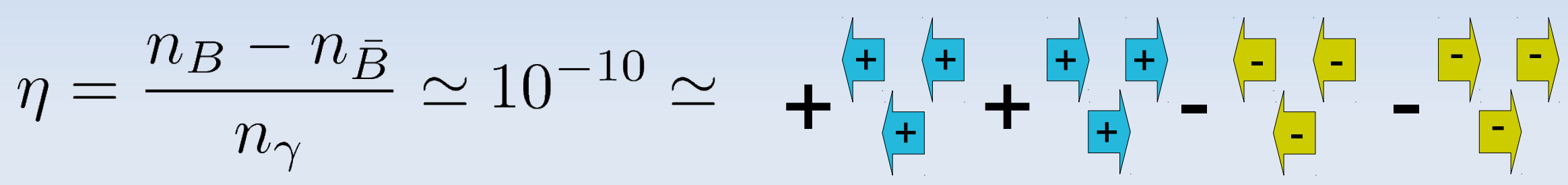

The main ingredients for viable baryogenesis are stated by the celebrated Sakharov conditions:

- B-number violation (baryon-number)

- C and CP violation (charge/parity)

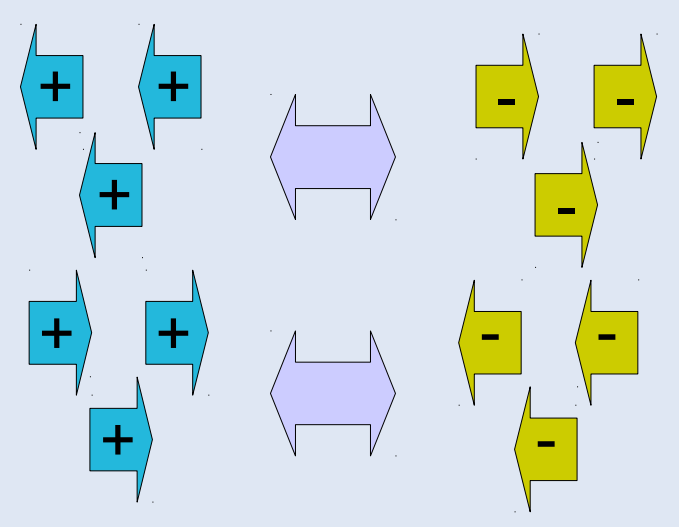

$$
n_{B} \leftrightarrow n_{\bar{B}}
$$

- out-of-equilibrium 


\section{Baryogenesis}

\section{[Sakharov '69]}

Baryogenesis aims at explaining the observed asymmetry between matter and antimatter abundances.

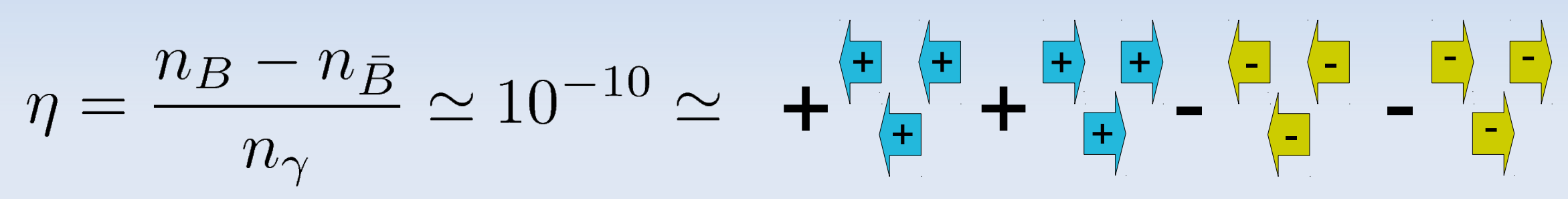

The main ingredients for viable baryogenesis are stated by the celebrated Sakharov conditions:

- B-number violation (baryon-number)

- $\mathrm{C}$ and $\mathrm{CP}$ violation (charge/parity)

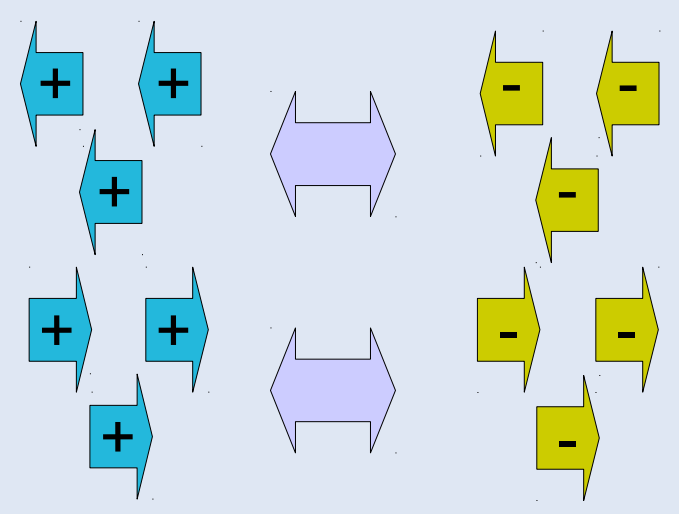

$$
n_{B} \leftrightarrow n_{\bar{B}}
$$

- out-of-equilibrium 


\section{Baryogenesis}

\section{[Sakharov '69]}

Baryogenesis aims at explaining the observed asymmetry between matter and antimatter abundances.

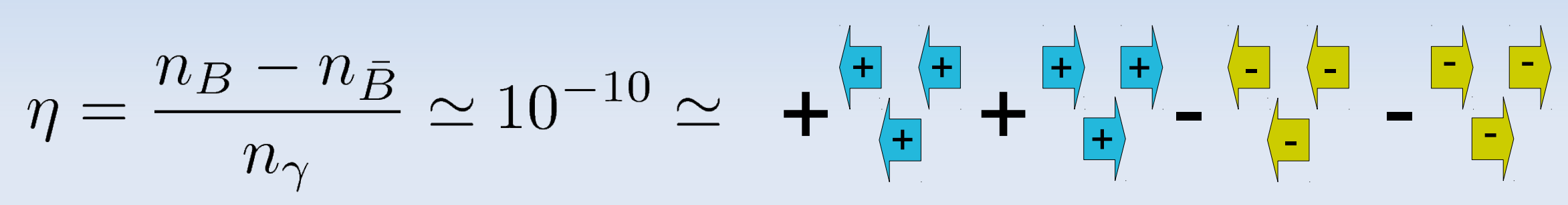

The main ingredients for viable baryogenesis are stated by the celebrated Sakharov conditions:

- B-number violation (baryon-number)

- $\mathrm{C}$ and $\mathrm{CP}$ violation (charge/parity)

$$
n=n(m / T)
$$

- out-of-equilibrium

$$
m=\bar{m}
$$

$$
n_{B}=n_{\bar{B}}
$$




\section{Baryogenesis}

\section{[Sakharov '69]}

Baryogenesis aims at explaining the observed asymmetry between matter and antimatter abundances.

$$
\eta=\frac{n_{B}-n_{\bar{B}}}{n_{\gamma}} \simeq 10^{-10}
$$

\section{SM @}

EW temp

C

B

$\mathrm{CP}$

eq

$$
B+L
$$

anomaly

Jarlskog invariant

expansion slow EW PT? sphaleron

beyond the SM physics essential 
The free energy (as a function of the Higgs vev) decides the nature of the phase transition:
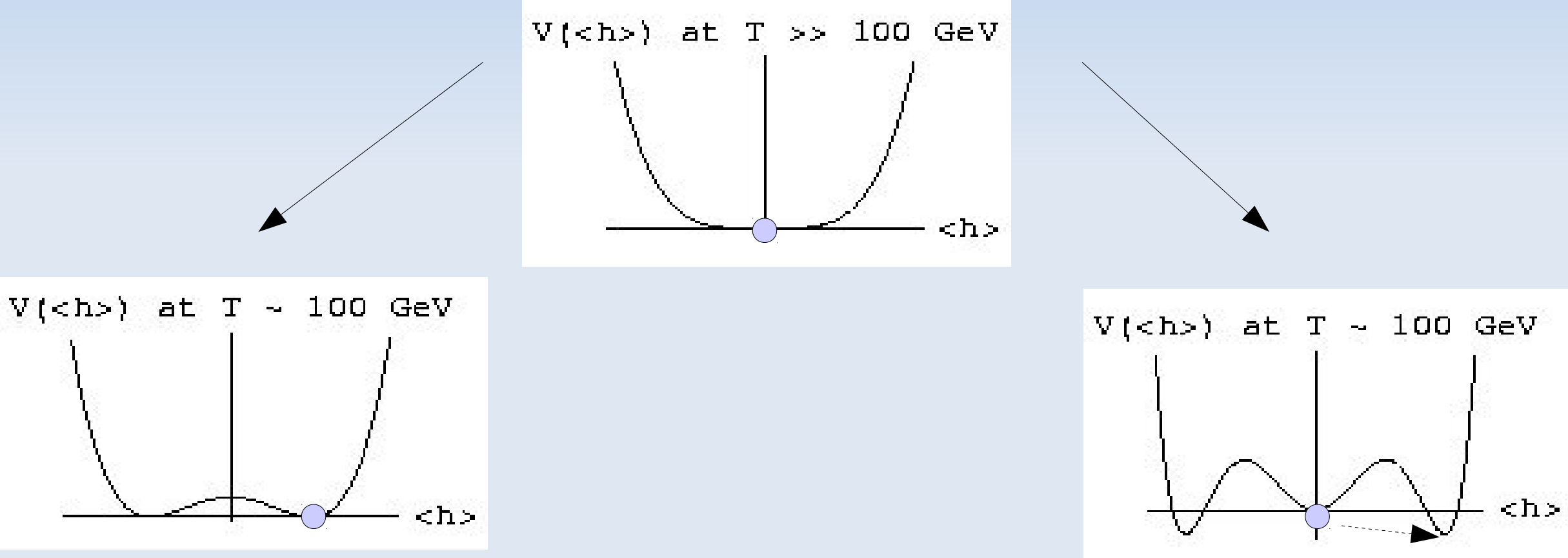

second-order crossover

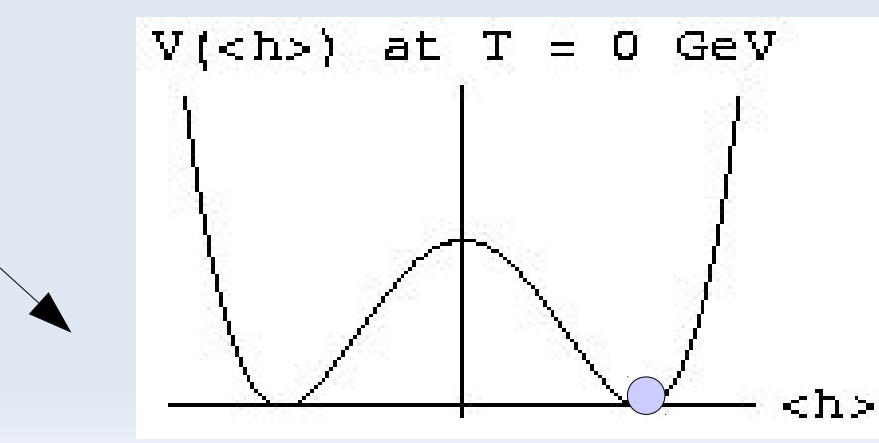

first-order 


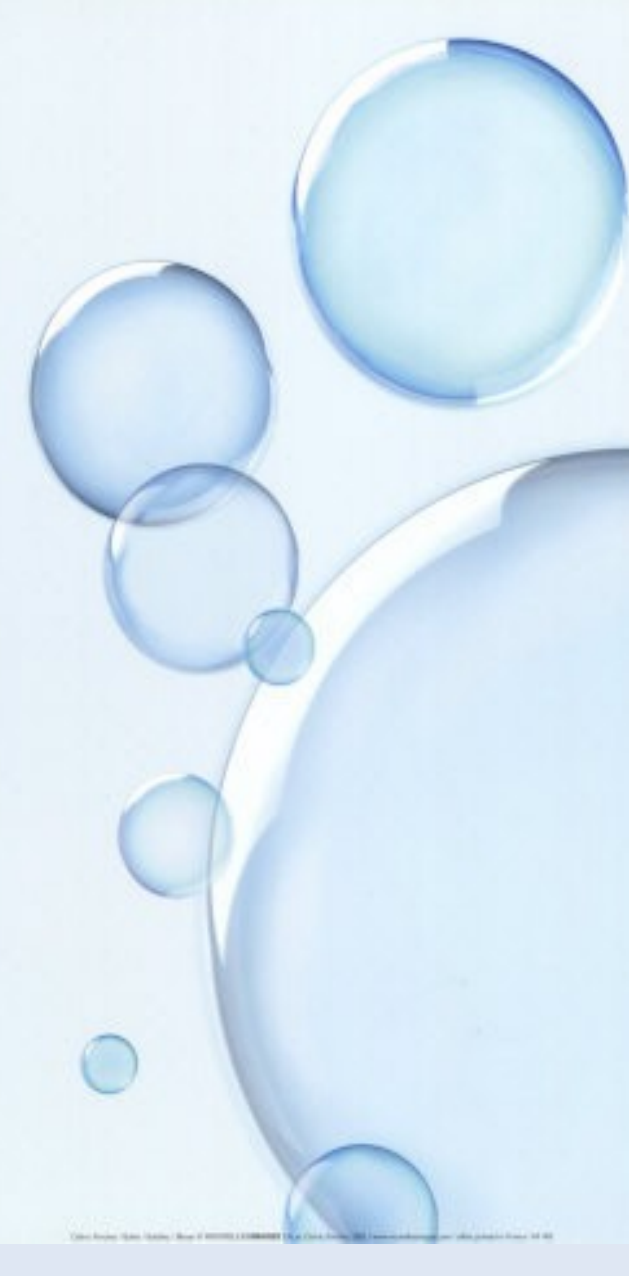

- first-order phase transitions proceed by bubble nucleations

- in case of the electroweak phase transition, the "Higgs bubble wall" separates the symmetric from the broken phase

- this is a violent process ( $v_{b}=O(1)$ ) that drives the plasma out-of-equilibrium

- bosons that are strongly coupled to the Higgs tend to make the phase transition stronger 


\section{Electroweak baryogenesis}

[Kuzmin, Rubakov, Shaposhnikov '85]

[Cohen, Kaplan, Nelson '93]

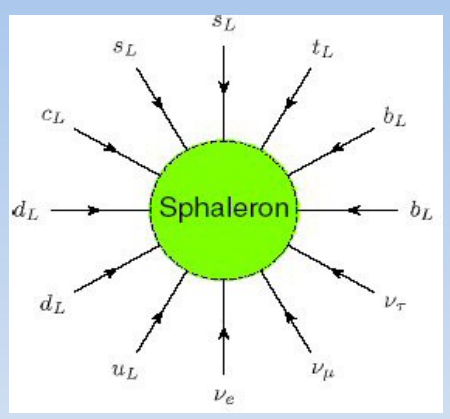

B $\mathrm{C}$

$\langle\phi\rangle$

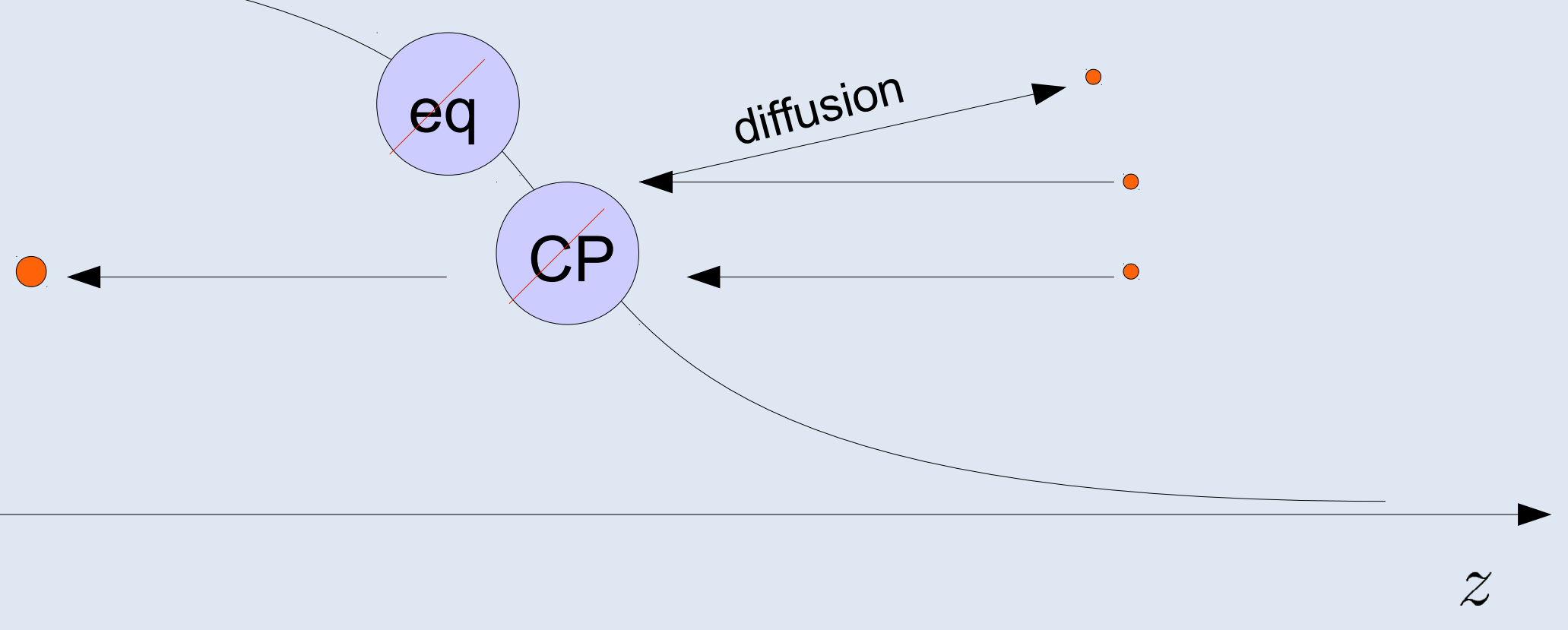




\section{Electroweak baryogenesis}

[Kuzmin, Rubakov, Shaposhnikov '85]

[Cohen, Kaplan, Nelson '93]

$\langle\phi\rangle$

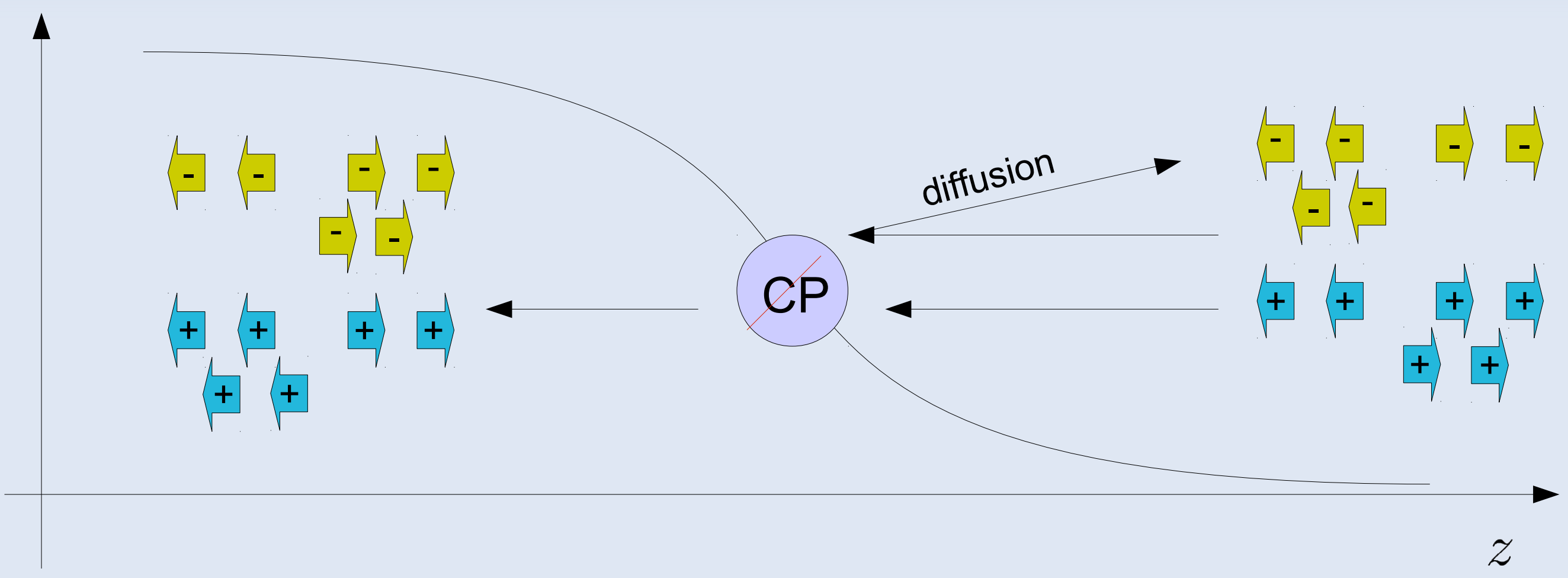




\section{Electroweak baryogenesis}

[Kuzmin, Rubakov, Shaposhnikov '85]

[Cohen, Kaplan, Nelson '93]

$\langle\phi\rangle$

B

$\Delta$

$\begin{array}{cc}-1 & -1 \\ -1 & -1\end{array}$

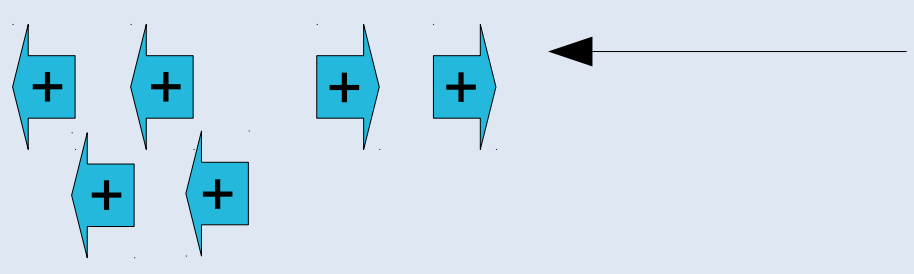

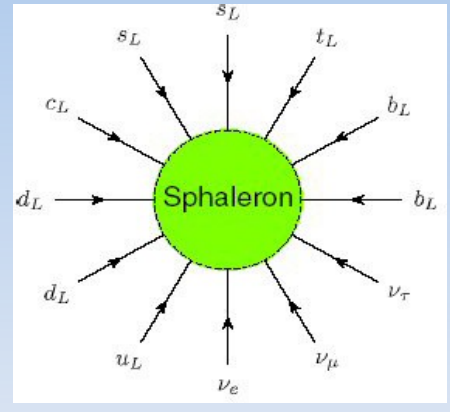

C

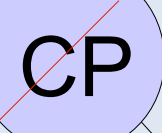

.

diffusion

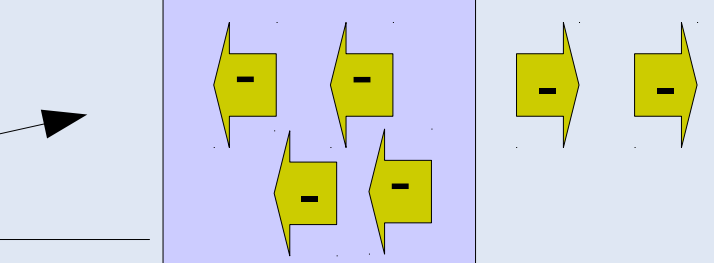

$+\mp$

++
++4 


\section{Electroweak baryogenesis}

[Kuzmin, Rubakov, Shaposhnikov '85]

[Cohen, Kaplan, Nelson '93]

$\langle\phi\rangle$

B

$\Delta$
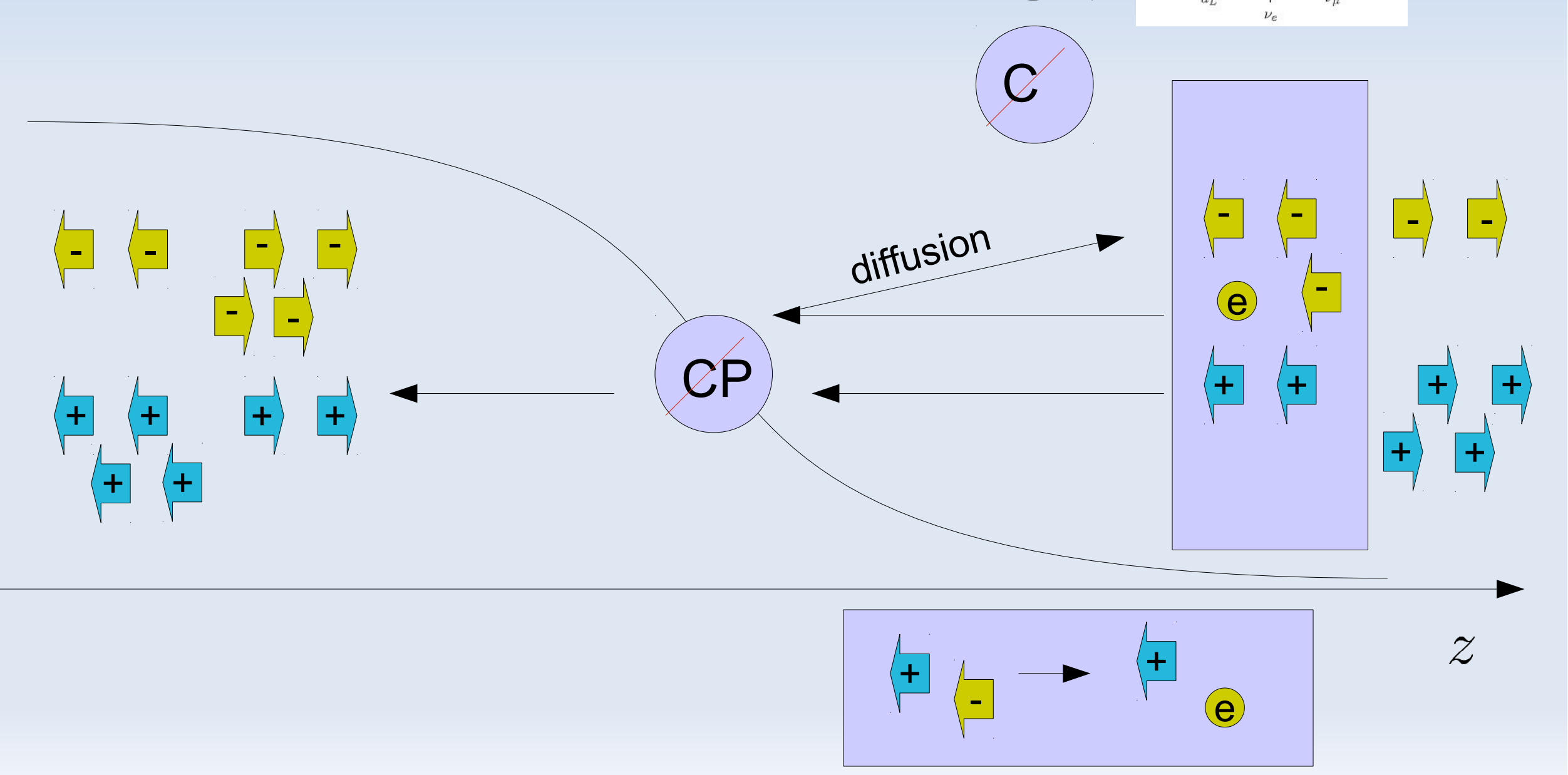


\section{Electroweak baryogenesis}

[Kuzmin, Rubakov, Shaposhnikov '85]

[Cohen, Kaplan, Nelson '93]

$\langle\phi\rangle$

\section{B}
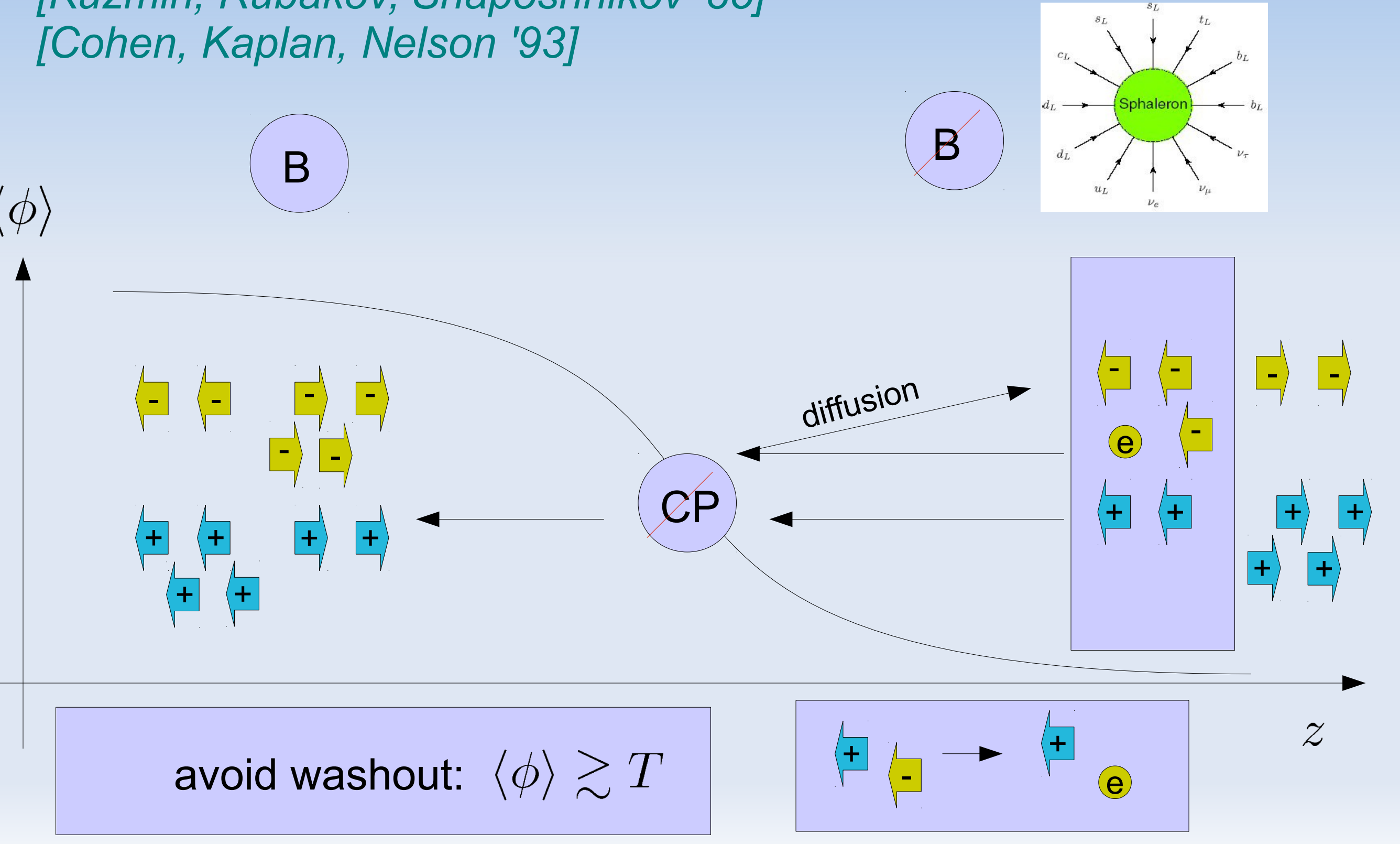


\section{Hierarchy problem}

$m_{\text {Higgs }}$

hierarchy problem

$m_{\text {Planck }}$

This indicates that there is some kind of new physics around the corner that did not appear in collider experiments yet

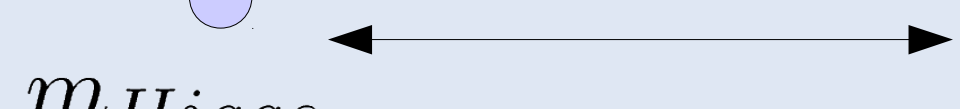

$m_{\text {Higgs }}$ small hierarchy problem
$\Lambda \Lambda_{\text {new }} \sim$
SUSY?

(warped) extra-dimensions strong coupling / technicolor? 


\section{Why is this interesting?}

The hierarchy problem indicates that there is some BSM physics at EW scales

Electroweak baryogenesis involves only physics at the electroweak scale that is accessible to collider experiments

Electroweak baryogenesis leads naturally to the observed baryon asymmetry

$$
\eta_{B} \sim \frac{\Gamma_{w s}}{l_{w} T^{2}} \delta_{C P} e^{-m_{\chi} / T} \sim 10^{-11}-10^{-9}
$$




\section{What are the challenges?}

[Kuzmin, Rubakov, Shaposhnikov '85]

[Cohen, Kaplan, Nelson '93]

sphaleron

(EW anomaly)

B

$\langle\phi\rangle$

statistical

quantum mechanics 
Technical details

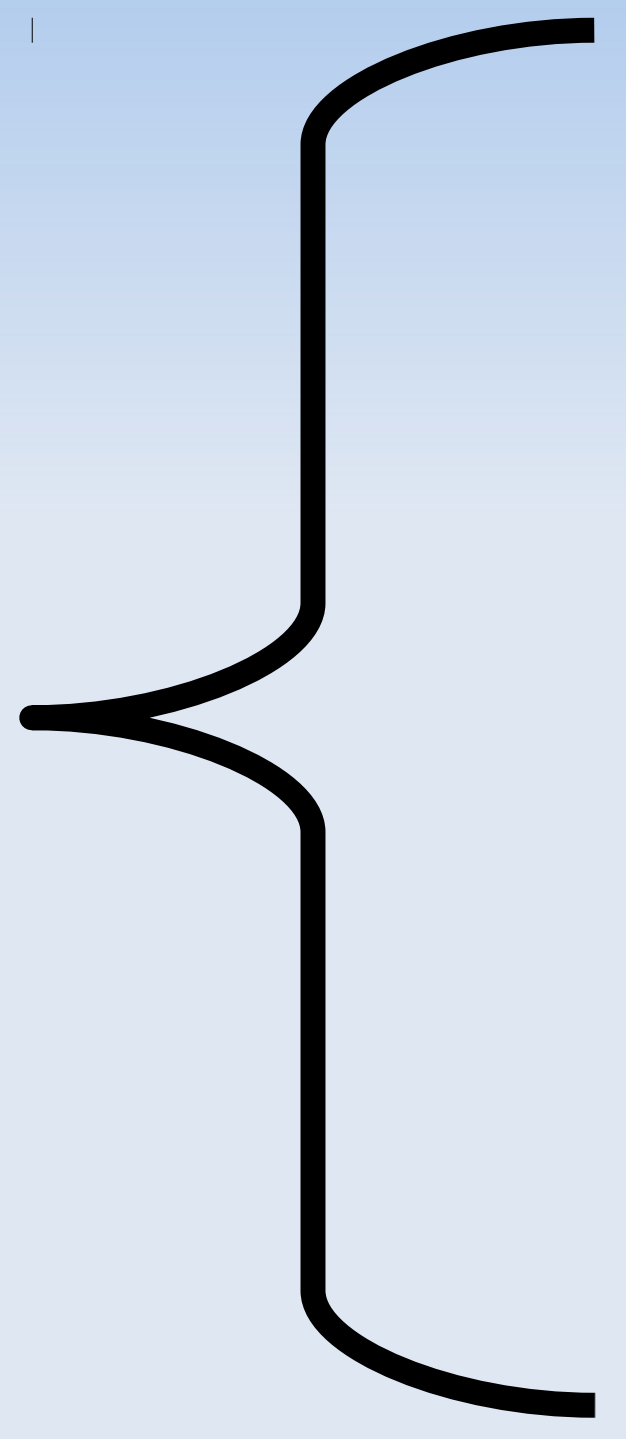




\section{Semi-classical reflection}

Many particles change their mass when passing into the Higgs bubble

$$
\begin{array}{cc}
m>0 & m=0 \\
\tilde{p}^{2}=m^{2} & p^{2}=0
\end{array}
$$

wall frame

$$
\tilde{E}=E \quad \text { and } \quad \tilde{p}_{z}^{2}=p_{z}^{2}-m^{2}
$$

reflection when $p_{z}<m$ 


\section{Transport equations}

In case of a statistical system with particle distribution function

$$
f\left(p_{z}, x, t\right)
$$

the change in momentum

$$
\tilde{E}=E \quad \text { and } \quad \tilde{p}_{z}^{2}=p_{z}^{2}-m^{2}
$$

translates into

$$
f\left(p_{z}, \text { left }\right)=f\left(\sqrt{p_{z}^{2}-m^{2}}, \text { right }\right)
$$

or

$$
\underbrace{p_{z} \partial_{z} f}_{\begin{array}{c}
\text { flow term } \\
\text { transport equation } \\
\text { of Boltzmann type }
\end{array}}+\underbrace{\frac{1}{2} \partial_{z} m(z)^{2} \partial_{p_{z}} f=0}_{\text {force }} \text { ? }
$$




\section{Kadanoff-Baym equations}

\section{[Kadanoff, Baym '61]}

In order to quantify electroweak baryogenesis one needs a formalism that includes quantum effects (CP violation) as well as statistical effects (diffusion/transport)

This is achieved by the Kadanoff-Baym equations that are a statistical generalisation of the Schwinger-Dyson equations of QFT (Schwinger-Keldysh formalism)

$$
\left(\square+m^{2}+\Sigma\right) G(x, y)=\delta(x-y)
$$

Formally the equation looks like SD, but

- The 2-point function depends on $x$ and y seperately

$\rightarrow X$ and $p$ in Fourier (Wigner) space

- There is an additional $2 \times 2$ structure from the in-in-formalism 


\section{Kadanoff-Baym equations}

In Wigner space this leads to the Moyal star product

$$
\left(p^{2}-m(X)^{2}-\Sigma\right) e^{i \diamond} G(X, p)=\mathbf{1}
$$

With $\diamond=\overleftarrow{\partial_{X}} \overrightarrow{\partial_{p}}-\overleftarrow{\partial_{p}} \overrightarrow{\partial_{X}}$

$$
X=\frac{x+y}{2}
$$

One of the Green functions (Wightman function) encodes the particle distribution function and is in equilibrium given by

$$
G_{e q}^{<}(X, p)=2 \pi i f^{e q}(p, X) \delta\left(p^{2}-m^{2}\right)
$$

and

$$
f^{e q}=\frac{1}{e^{p_{0} / T} \pm 1}
$$




\section{One fermion flavor}

For one fermion flavor with a space-time dependent mass, the Kadanoff-Baym equation reads

$$
\left(\not p-m P_{L}-m^{*} P_{R}-\Sigma^{R}\right) e^{i \diamond} G^{<}=\text {collisions }
$$

In order to do progress analytically, one typically uses several approximations/techniques:

1) Neglect most interactions: $\quad \Sigma^{R} \rightarrow 0$

2) Planar approximation (z): $\hat{S} \propto\left(p_{0} \gamma_{0}-p_{\perp} \cdot \gamma\right) \gamma_{3} \gamma_{5}$

3) Gradient expansion: $\quad \diamond \sim \partial_{x} \partial_{p} \sim(L T)^{-1} \ll 1$ 


\section{One fermion flavor}

For one fermion flavor with a space-time dependent mass, an expansion in gradients leads to the equations

$$
\left(p^{2}-m^{2}+\cdots\right) G^{<}=0
$$

and with $m(z)=|m(z)| e^{i \theta(z)}$ and spins $\mathbf{s}$

$$
\left(p \cdot \partial_{X}+\frac{1}{2}\left(m^{2}\right)^{\prime} \partial_{p}+\frac{s}{2 p_{z}}\left(m^{2} \theta^{\prime}\right)^{\prime} \partial_{p}\right) f_{s}=\text { collisions }
$$

$m\left(\partial_{t} \dot{+} \vec{v} \cdot \nabla\right) \quad$ forces

[Cline, Joyce, Kainulainen '00]

$\mathrm{CP}$

[Kainulainen, Prokopec, Schmidt, Weinstock '02] decays scatterings 
Technical details

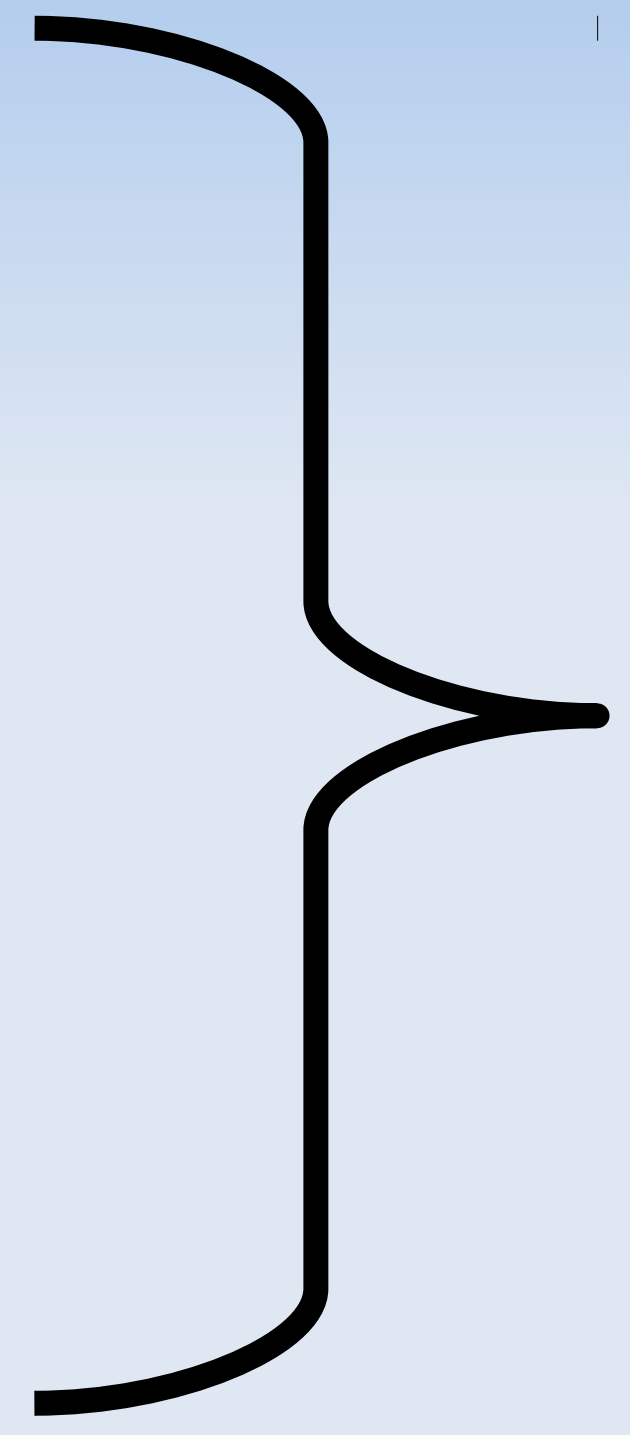




\section{Ingredients}

1 eq Strong first-order electroweak phase transition $\phi>T$

Some fermion species that changes its

2 CP mass in a CP violating way during the electroweak phase transition (and prefereably charged under $S U(2)_{L}$ ) 


\section{Summary}

$\left(p \cdot \partial_{X}+\frac{1}{2}\left(m^{2}\right)^{\prime} \partial_{p}+\frac{s}{2 p_{z}}\left(m^{2} \theta^{\prime}\right)^{\prime} \partial_{p}\right) f_{s}=$ collisions

$\langle\phi\rangle$

B

$$
m=|m(X)| e^{i \theta(X)}
$$

$\mathrm{CP}$

B 


\section{Outline}

\section{Introduction}

MSSM

Composite Higgs 
In the minimal supersymmetric standard model, the leading

$\mathrm{CP} \quad \mathrm{CP}$ violation comes from the mass matrix of the

$$
m=\left(\begin{array}{cc}
M_{2} & g v_{1}(X) \\
g v_{2}(X) & \mu_{c} e^{i \varphi}
\end{array}\right) \quad \varphi \neq 0
$$

The strength of the electroweak phase transition

is reduced by larger Higgs masses is enhanced by bosonic degrees of freedom that couple strongly to the Higgs - stops important

$$
m_{\text {stop }} \lesssim m_{\text {top }}
$$




\section{Several flavours}

In the most prominent case (MSSM), CP violation results from mixing effects between different flavors.

[Careno, Moreno, Quiros, Seco, Wagner '00]

In this case, new CP-violating sources occur

$$
\text { forces } \ni-\frac{1}{4}\left\{m^{2 \prime}, \partial_{p} \cdot\right\}+\frac{i}{16}\left[m^{2 \prime \prime}, \partial_{p}^{2} .\right]
$$

but also additional complications arise due to flavor oscillations

$$
\begin{gathered}
\left(p \cdot \partial_{X}+\frac{i}{2}\left[m^{2}, .\right]+\text { forces }\right) f_{s}=\text { collisions } \\
e^{i \frac{\Delta m^{2}}{p_{z}} z} \longrightarrow \text { gradient expansion? }
\end{gathered}
$$


Assuming a strong first-order phase transition, the baryon assymetry depends mostly on the chargino mass and the $\mathrm{CP}$ violation in the chargino sector

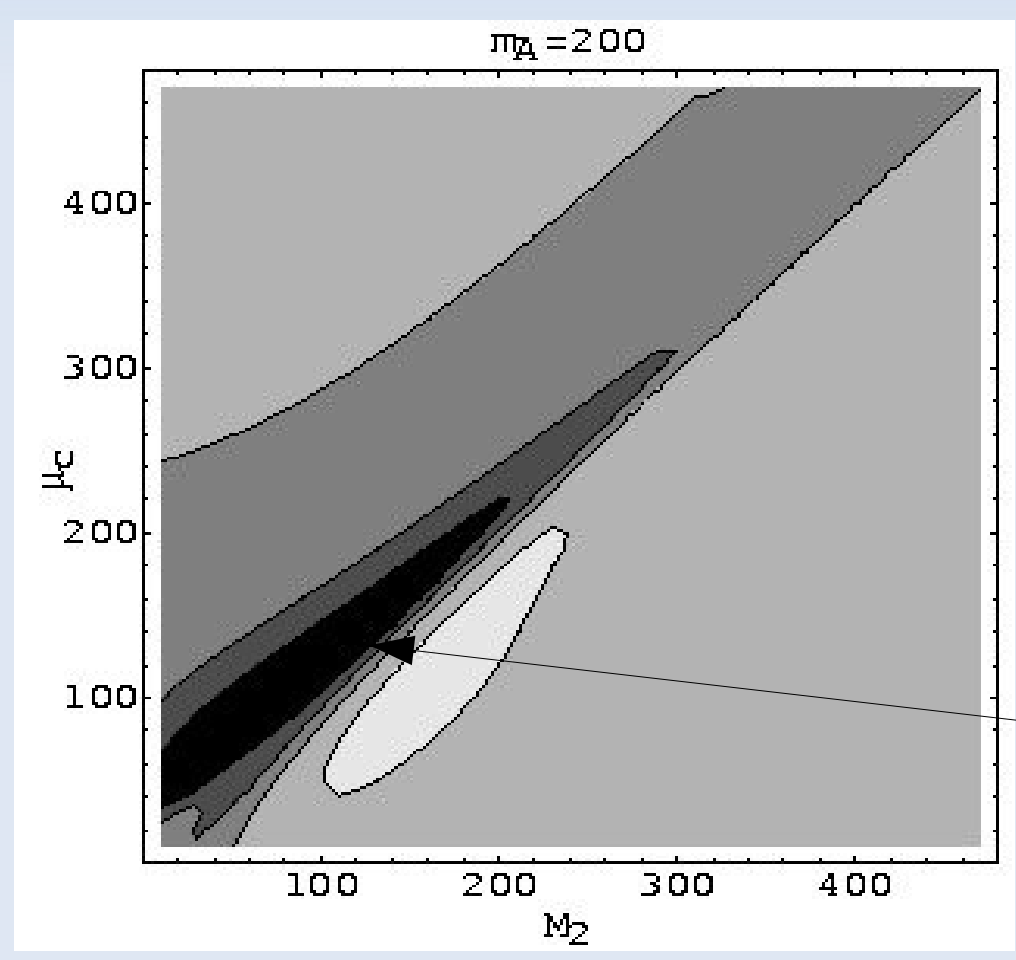

$$
\begin{gathered}
m=\left(\begin{array}{cc}
M_{2} & g v_{1}(X) \\
g v_{2}(X) & \mu_{c} e^{i \varphi}
\end{array}\right) \\
\varphi=\frac{\pi}{2} \\
\frac{\eta_{\max }}{\eta_{\text {observed }}} \simeq 5
\end{gathered}
$$

[TK, Prokopec, Schmidt, Seco '05] 
In the minimal supersymmetric standard model, the leading $\mathrm{CP}$ violation comes from the mass matrix of the charginos

$$
m=\left(\begin{array}{cc}
M_{2} & g v_{1}(X) \\
g v_{2}(X) & \mu_{c} e^{i \varphi}
\end{array}\right)
$$

Flavor mixing?

Resonances?

Higgs resummation?

Flavor oscillations?

Higgs transport?

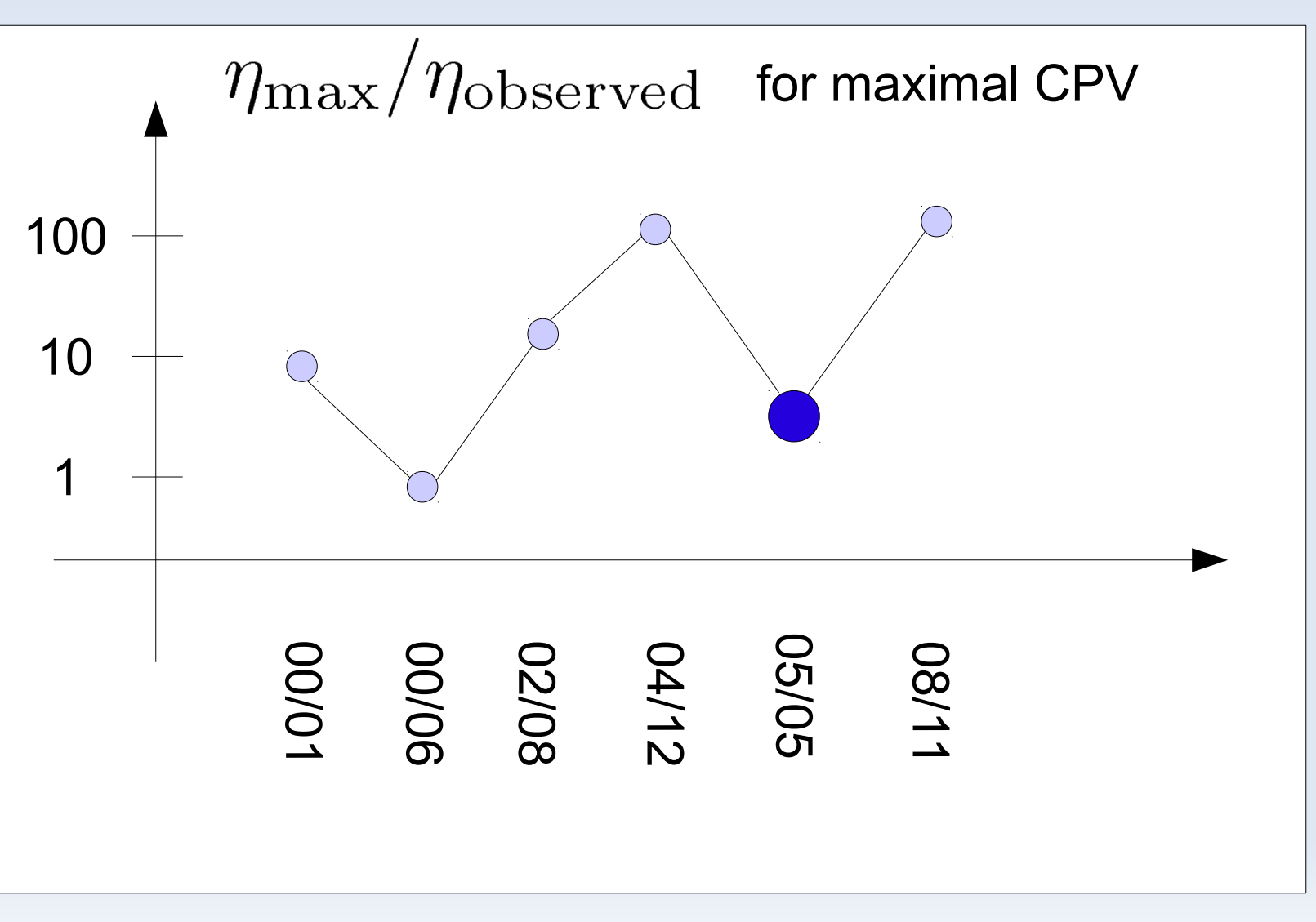




\section{Electric dipole moments}

Even if all sfermions are heavy, there are two-loop (Barr-Zee) contributions from the chargino to the EDM of the electron and the neutron
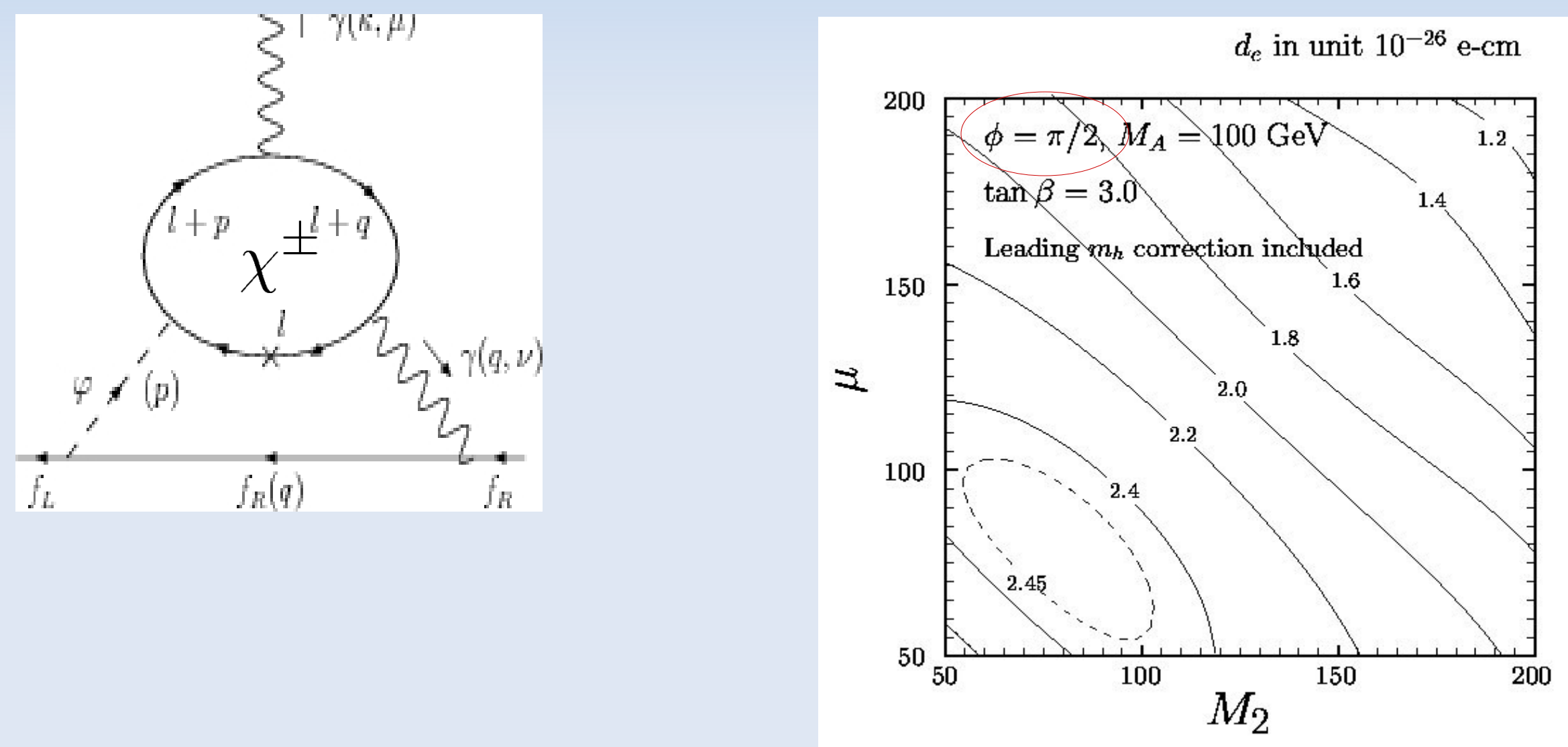

[Chang, Chang, Keung '02] 


\section{EDMs}

Even if all sfermions are heavy, there are two-loop (Barr-Zee) contributions from the chargino to the EDM of the electron and the neutron

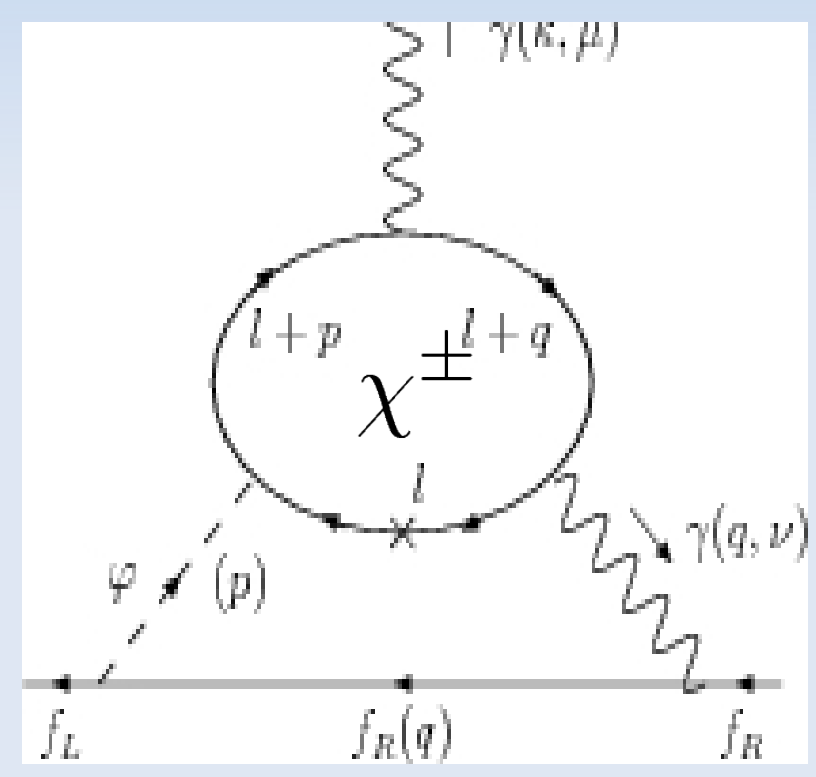

$\sin \varphi<?$

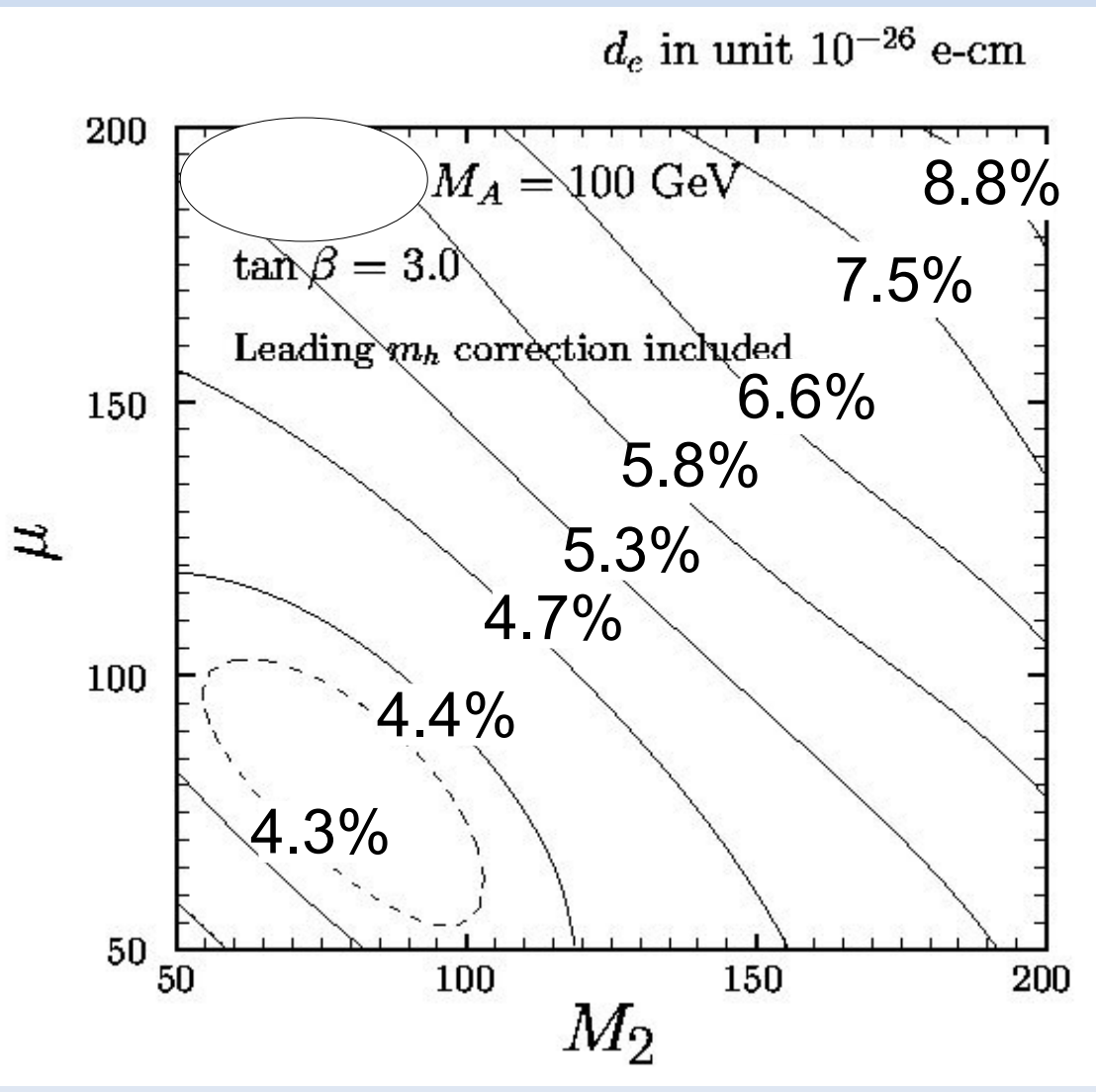

[Chang, Chang, Keung '02]

[Hudson et al. '11]

$d_{e}<1.05 \times 10^{-27} e \mathrm{~cm}$ 


\section{MSSM baryogenesis}

[Hudson et al. '11]

The EDM constraints translate into $\eta_{\max } / \eta_{\text {observed }} \gtrsim 23$

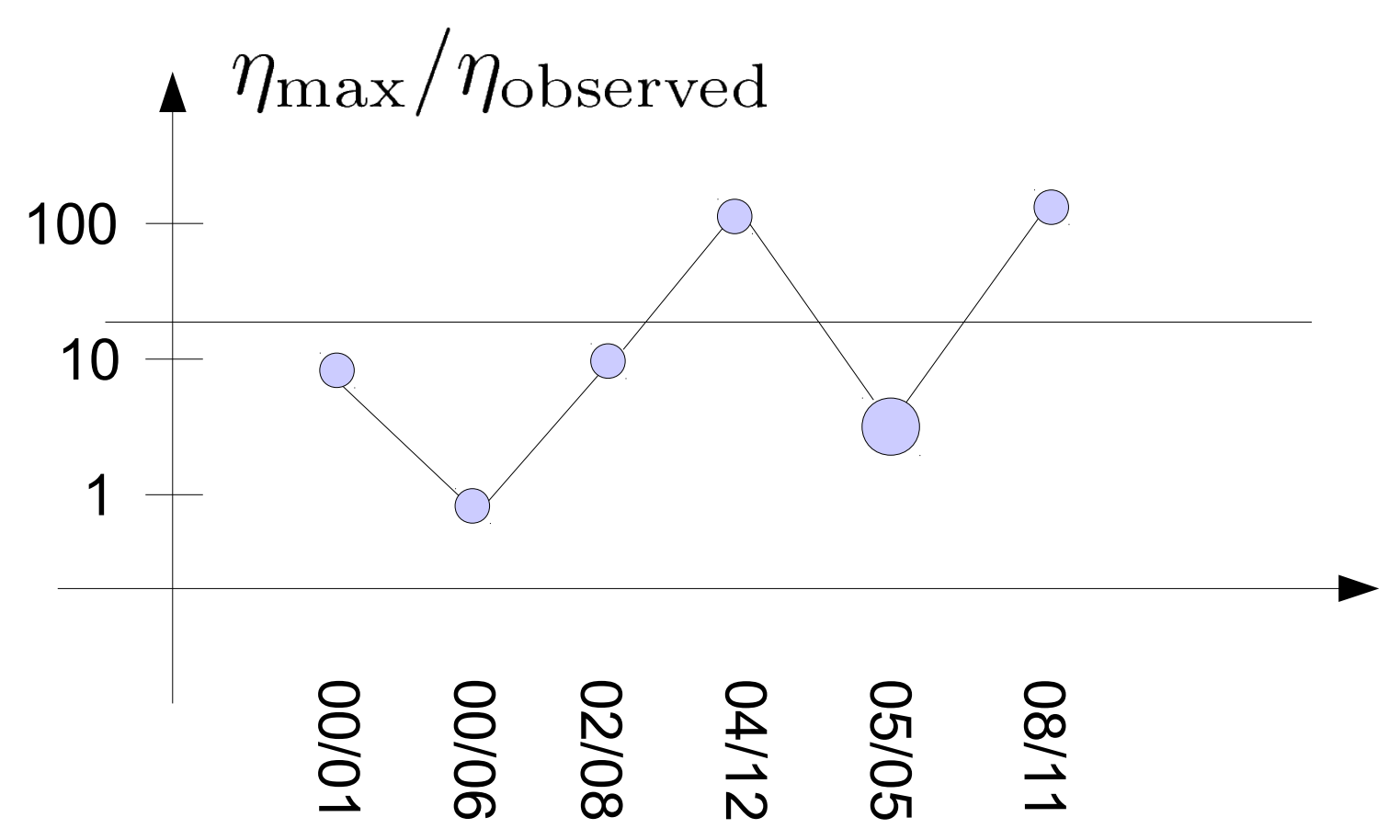




\section{Chargino driven MSSM baryogenesis ruled out}

[Baron et al. '13]

The EDM constraints translate into $\eta_{\max } / \eta_{\text {observed }} \gtrsim 250$

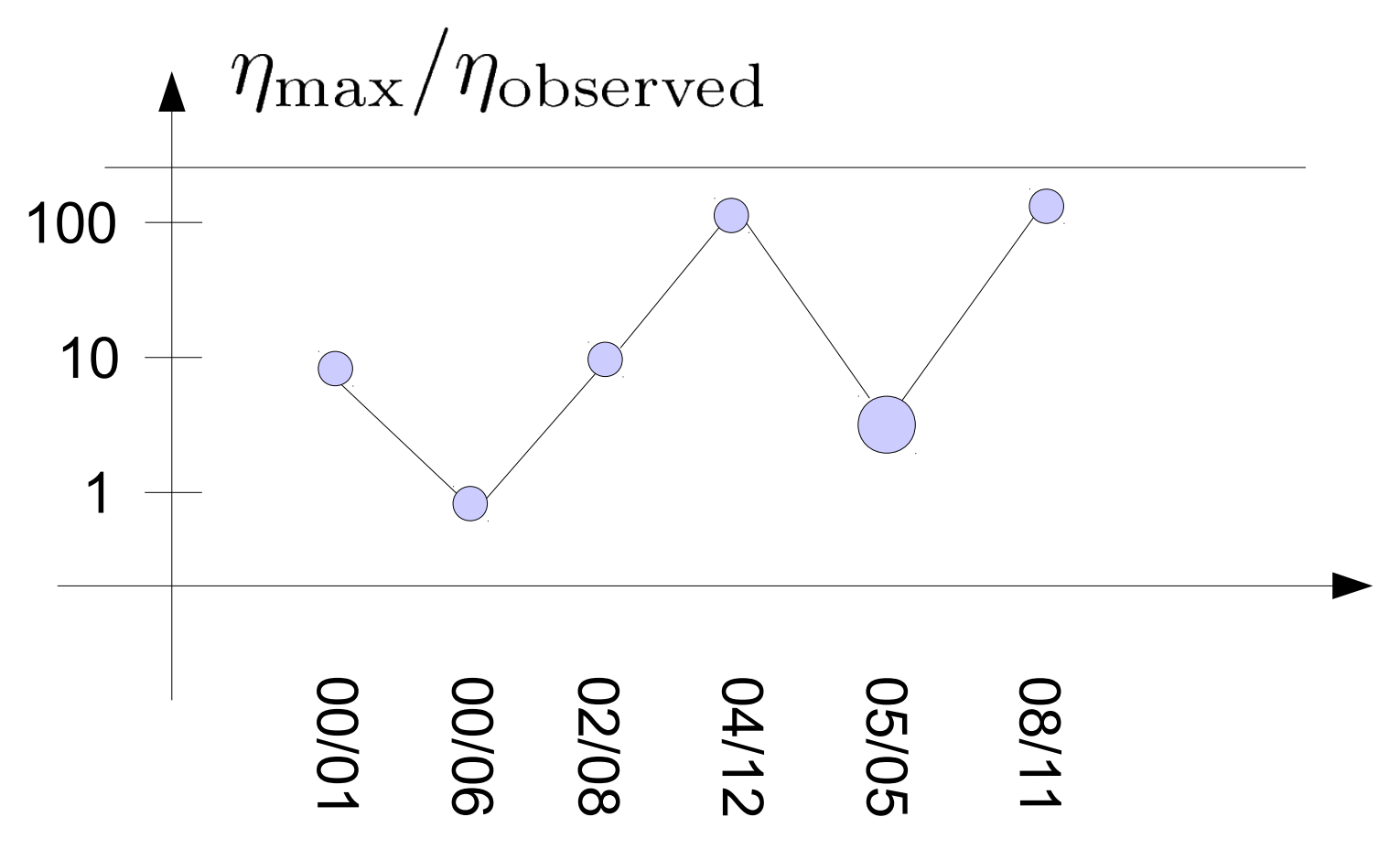

Caveat: EDM bounds avoided by using binos

[Li, Profumo, Ramsey-Musolf '08] 
MSSM phase transition after LEP: very heavy left-handed stop required

$$
m_{\text {stop }, \mathrm{L}} \sim 500 \mathrm{TeV}
$$

$\frac{\phi_{c}}{T_{c}}>1.0$

$m_{\text {stop, R }}$

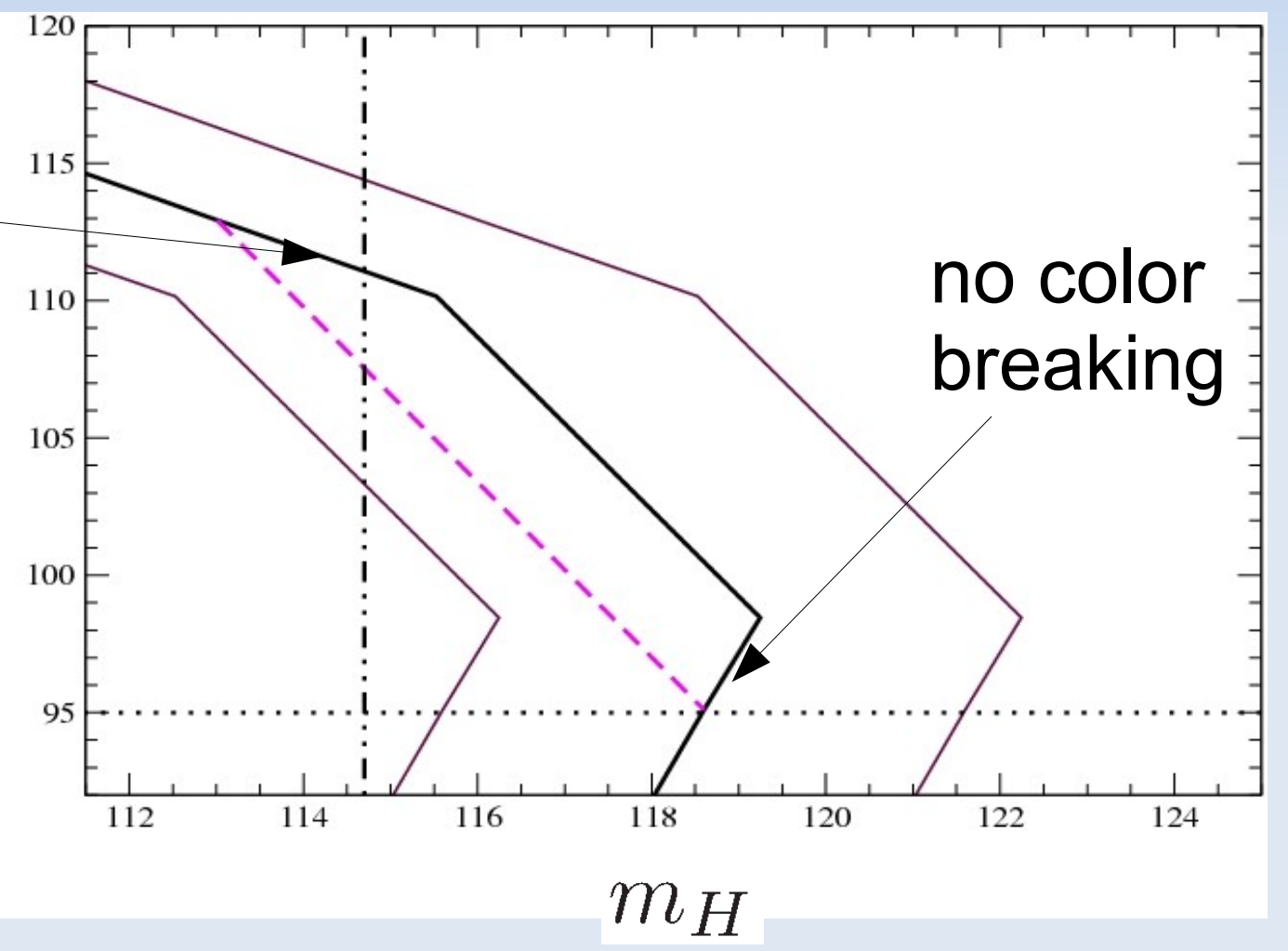

$m_{\text {stop, } \mathrm{R}} \lesssim 110 \mathrm{GeV} \quad m_{\text {stop }, \mathrm{L}} \gtrsim 30 \mathrm{TeV}$

[Carena, Nardini, Quiros, Wagner '08] 
MSSM phase transition after LEP: very heavy left-handed stop required

$\frac{\phi_{c}}{T_{c}}>1.0$

$m_{\text {stop, R }}$

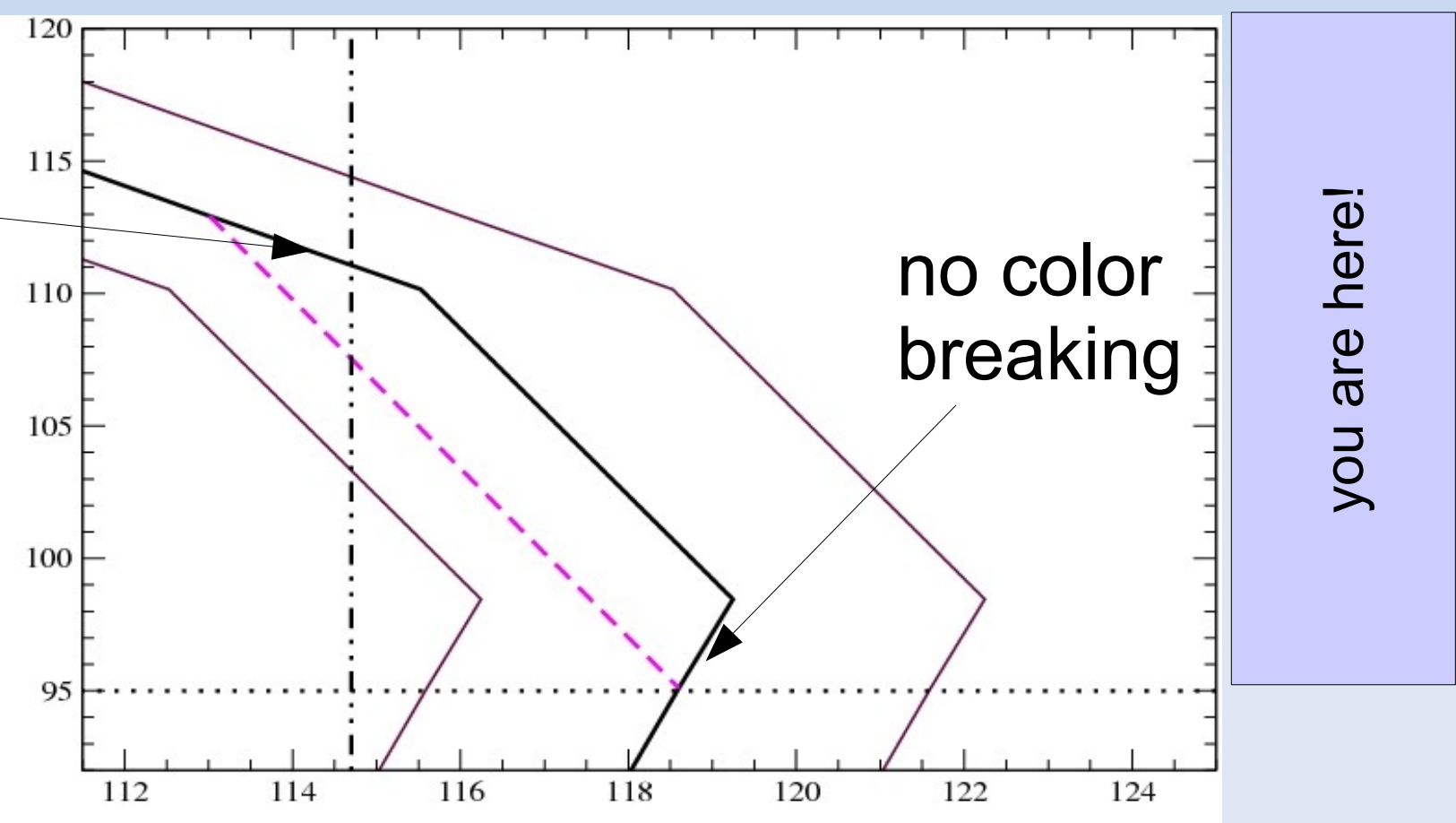

$m_{H}$

- $m_{\text {stop }, \mathrm{L}} \gtrsim 10^{6} \mathrm{TeV}$

[Carena, Nardini, Quiros, Wagner '08]

and hide right-handed stop from LHC 


\section{Conclusions MSSM}

Electroweak baryogenesis in SUSY models is yet, but with current experimental constraints it is a reather contrived

\section{scheme}

Light $(<200 \mathrm{GeV})$ and almost mass degenerate charginos or binos

$\mathrm{CP}$ violation for charginos that in the most optimistic cases is at the verge of beeing seen in EDM experiments (caveat: binos)

very specific spectrum required for a strong first-order phase transition (or extended Higgs sector?) 


\section{Outline}

\section{Introduction}

MSSM

Composite Higgs 


\section{Composite Higgs models}

The Higgs could be a Pseudo-Goldstone boson of a broken global symmetry

$$
\text { QCD: } \quad \frac{S U(2)_{L} \times S U(2)_{R}}{S U(2)_{V}} \rightarrow 3 \pi
$$

The broken symmetry will determine the light degrees of freedom and their quantum numbers

but also

$$
\frac{S O(5)}{S O(4)} \rightarrow H
$$

$$
\frac{S O(6)}{S O(5)} \rightarrow H+S \quad \frac{S O(6)}{S O(4) \times S O(2)} \rightarrow 2 H
$$

[Kaplan, Georgi '84] 


\section{Holographic techniques in composite Higgs models}

Lately, this old idea underwent a renaissance due to holographic models to determine some quantity of the strongly coupled theory (like the Higgs potential) in a 5D setup.

[Contino, Nomura, Pomarol '03]

[Agashe, Contino, Pomarol '04]

5D GIM mechanism for flavor problems

oblique parameters better than in technicolor many parameters and a certain arbitrariness in the scalar sector 


\section{Ingredients}

Two ingredients of baryogenesis are missing in the Standard Model. These are provided in models that have an additional singlet in the low energy description

Strong first-order electroweak $\quad V(s, h)$ phase transition

$\mathrm{CP}$ violation

CP from dimension-five operators

$\mathcal{L} \ni y_{t} \bar{\psi}_{Q} H \psi_{t}+\frac{\tilde{y}_{t}}{f} S \bar{\psi}_{Q} H \psi_{t}+$ h.c. 
The construction of a potential barrier and hence first-order phase transitions are easily achieved in extended scalar sectors:

$$
\begin{aligned}
V(h, s) & =\frac{\lambda}{4}\left(h^{2}-v^{2}\right)^{2} \\
& +m_{s}^{2} s^{2}+a_{s} s^{3}+\lambda_{s} s^{4} \\
& +a_{m} s h^{2}+\lambda_{m} s^{2} h^{2}
\end{aligned}
$$

For example consider deformations of the $\mathbb{Z}_{2}$ - symmetric "super-Mexican-hat"

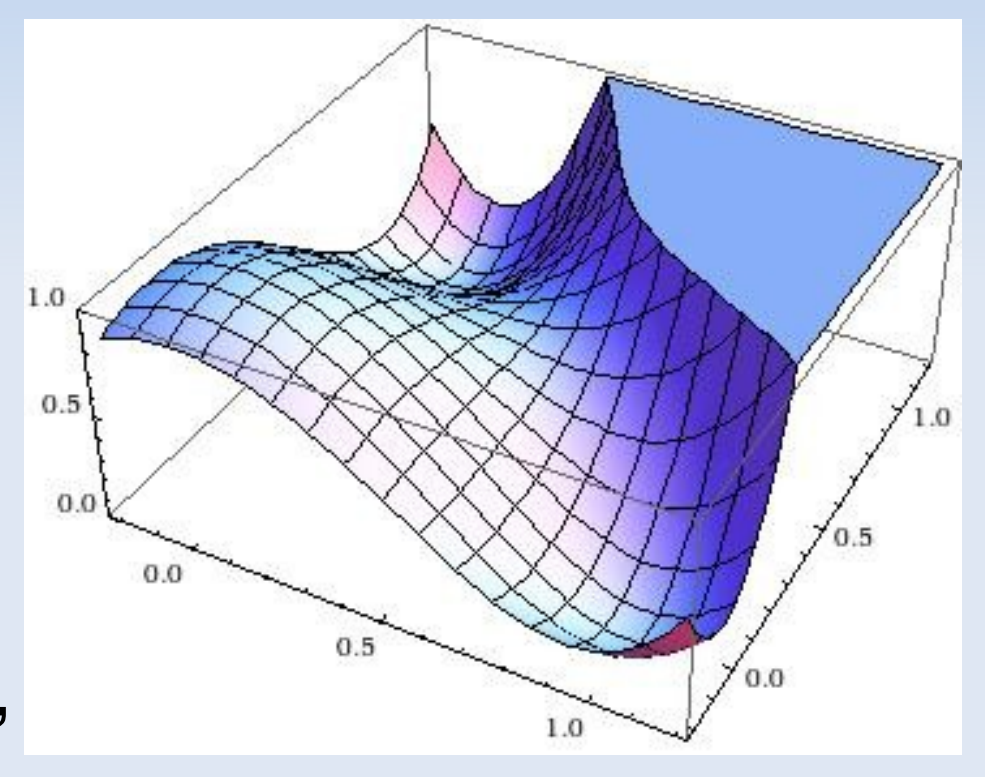

$$
V(s, h)=\frac{\lambda}{4}\left(h^{2}+s^{2} / \alpha^{2}-v^{2}\right)^{2}+\lambda_{m} h^{2} s^{2}
$$

that has a phase transition

$$
(h, s)=(0, \alpha v) \rightarrow(h, s)=(v, 0)
$$




$$
\mathcal{L} \ni y_{t} \bar{\psi}_{t} H \psi_{t}+\frac{\tilde{y}_{t}}{f} S \bar{\psi}_{t} H \psi_{t}
$$

During the phase transition this leads to a top mass of the form

$$
m_{t}=\left|m_{t}\right| e^{i \theta_{t}}=\frac{y_{t} h}{\sqrt{2}}\left(1+\frac{\tilde{y}_{t}}{y_{t}} \frac{s}{f}\right)
$$

So, the complex phase during the phase transition behaves as

$$
\theta_{t} \simeq \frac{\Im\left(y_{t} \tilde{y}_{t}^{*}\right)}{y_{t} y_{t}^{*}} \frac{s}{f}
$$

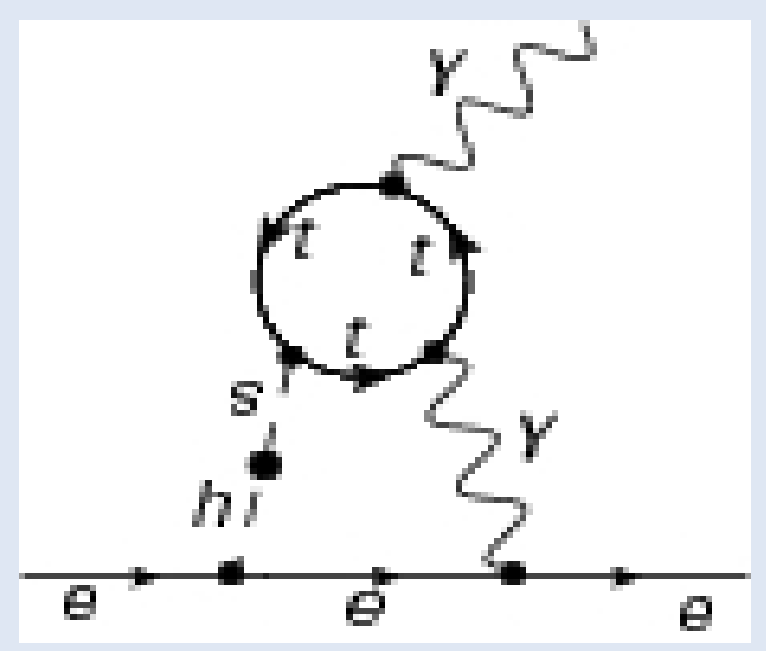

This is a one flavor system and the BAU can be reliably determined with the semi-classical force approach. 


\section{Baryogenesis}

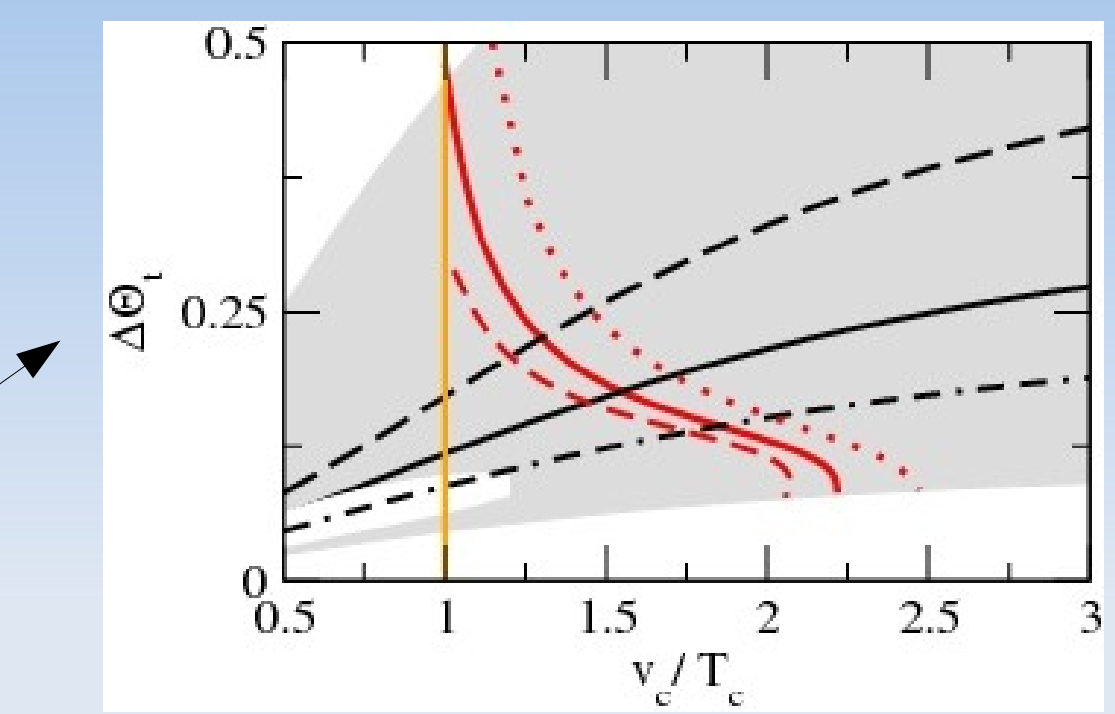

$$
m_{s}=130 \mathrm{GeV}
$$

$$
\Delta \theta_{t} \gtrsim 0.15
$$

strength of $\mathrm{CP}$ violation
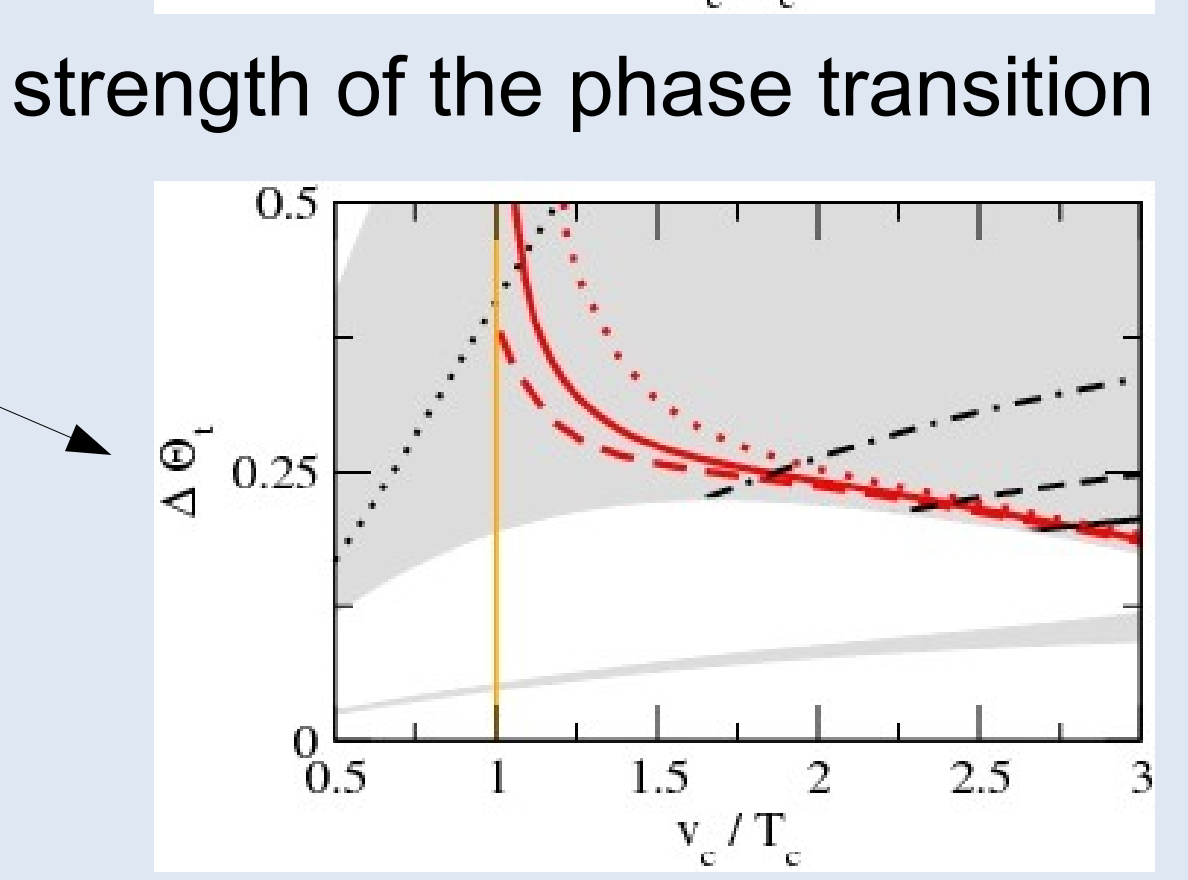

$\Delta \theta_{t} \simeq \frac{\Im\left(y_{t} \tilde{y}_{t}^{*}\right)}{y_{t} y_{t}^{*}} \frac{\Delta s}{f}$

$$
\begin{gathered}
m_{s}=80 \mathrm{GeV} \\
\Delta \theta_{t} \gtrsim 0.25
\end{gathered}
$$

[Espinosa, Gripaios, TK, Riva '11] 


\section{Signals}

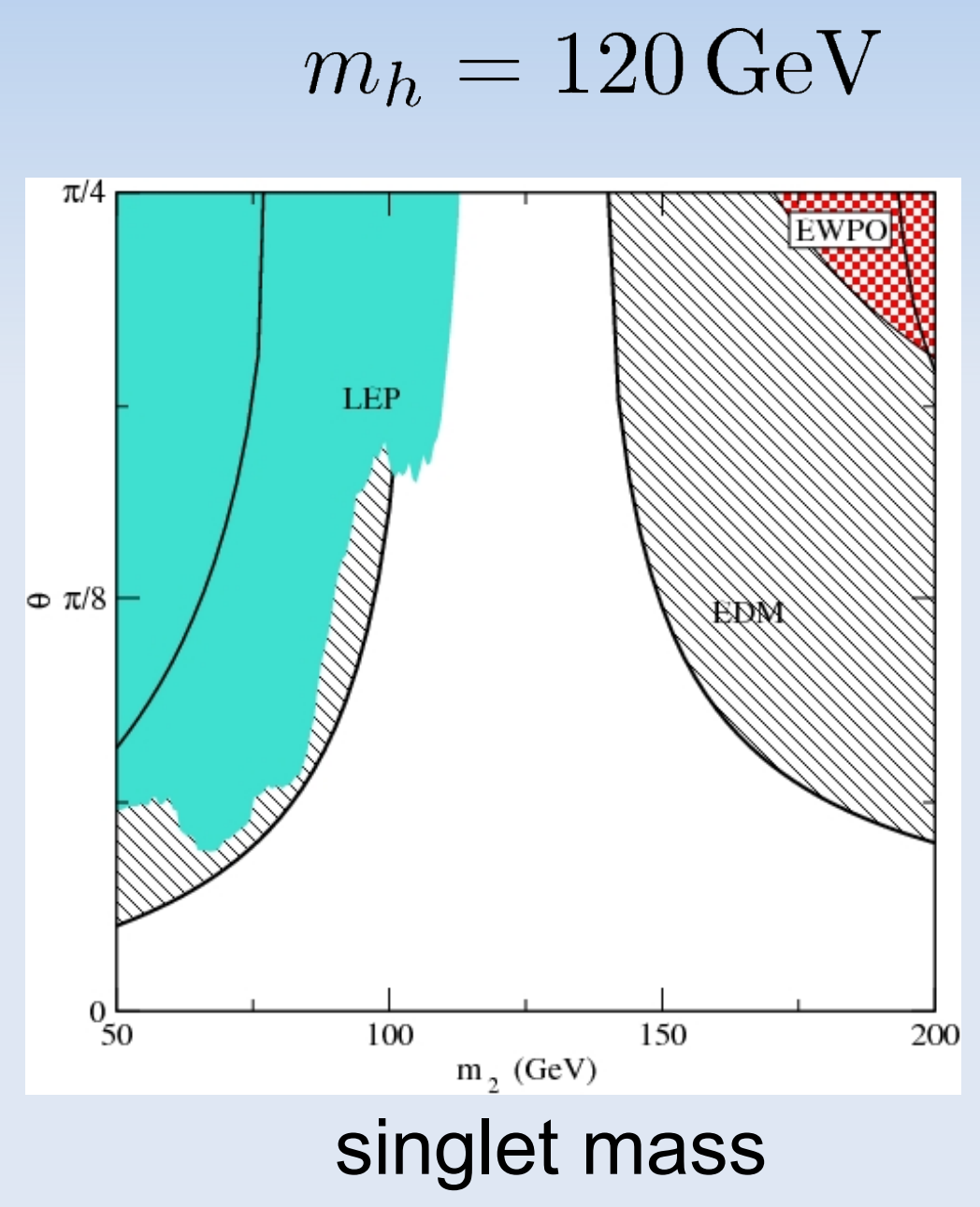

[Espinosa, Gripaios, TK, Riva '11]

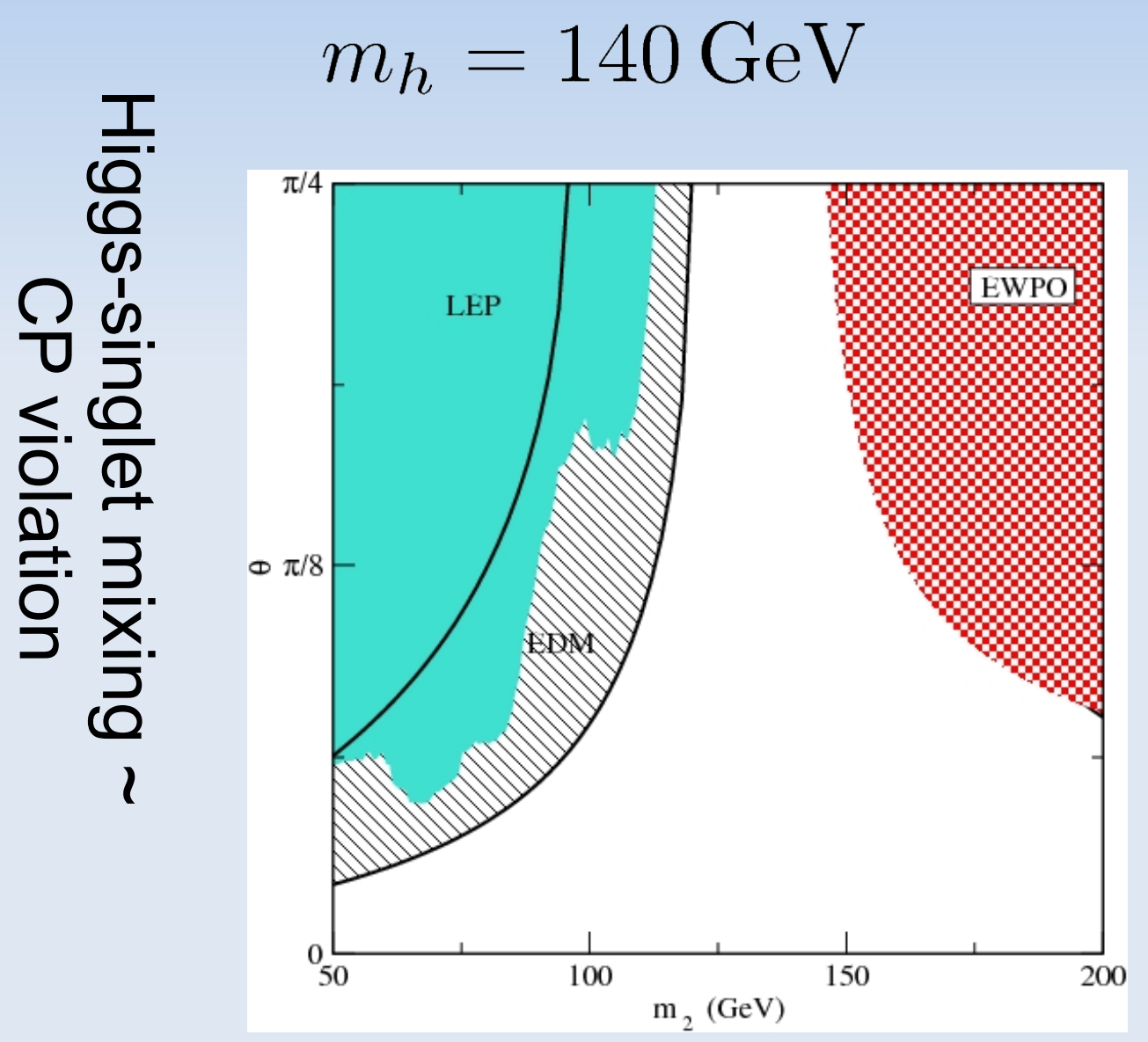

$$
\frac{\Im\left(y_{t} \tilde{y}_{t}^{*}\right)}{y_{t} y_{t}^{*}} \frac{1}{f}=(500 \mathrm{GeV})^{-1}
$$




\section{Conclusions composite Higgs}

Baryogenesis in composite Higgs models is generically possible if the sector of pseudo-Goldstone bosons is non-minimal.

In the case of a scalar extension of the low energy theory, this leads to

rich phenomenology

traces of $\mathrm{CP}$ violation in terms of EDMs

no $\mathbb{Z}_{2} \quad$ (Higgs-singlet mixing; singlet is not DM) 


\section{Conclusions}

Electroweak baryognesis is still a compelling framework to explain the observed baryon asymmetry.

Higgs found

No EDMs

No minimal SUSY

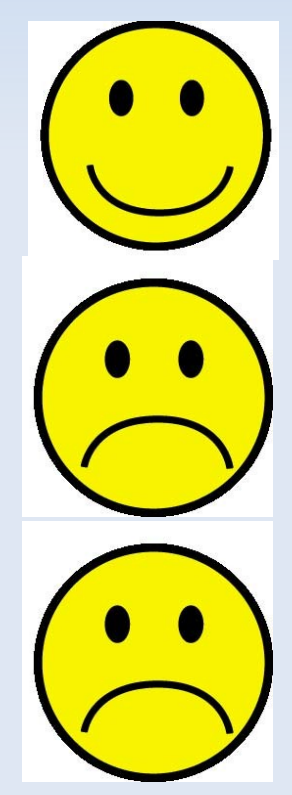




\section{Conclusions}

Electroweak baryognesis is still a compelling framework to explain the observed baryon asymmetry.

Higgs found

No EDMs

No minimal SUSY

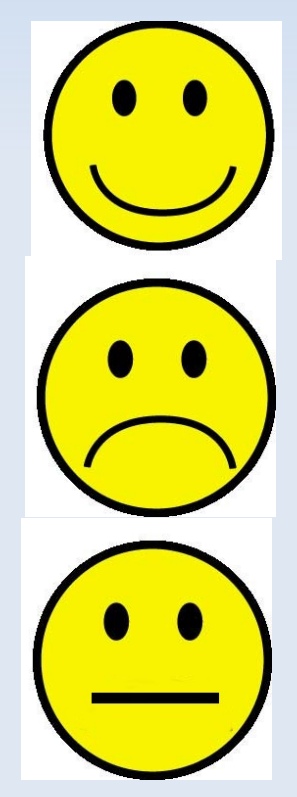

